\title{
EVALUACIÓN DE TRES DIFERENTES \\ FERMENTACIONES DE BIOL, SOBRE DOS SISTEMAS DE COBERTURA INERTE, BAJO DOS DENSIDADES DE SIEMBRA EN EL ESTABLECIMIENTO DEL CULTIVO NO TRADICIONAL DE KA' A HE' E (Stevia rebaudiana) HIERBA DULCE EN LA ZONA DE SANTO DOMINGO DE LOS TSÁCHILAS.
}

\author{
Autores: \\ Luis Gusqui' \\ César Huisha' \\ Ángel Albán' \\ Eduardo Lema ${ }^{1}$
}





\section{Resumen}

La presente investigación está enfocada a evaluar el efecto de diferentes fermentaciones de biol bajo sistemas de cobertura inerte, sobre densidades de siembra en el establecimiento y rendimiento del cultivo no tradicional de la (Stevia rebaudiana). La investigación se efectuó en la Granja Experimental de la Carrera de Ingeniería Agropecuaria de la Universidad Tecnológica Equinoccial, Campus Santo Domingo. Se sembraron 13000 plantines de Stevia, utilizando dos distanciamientos de $70 \mathrm{~cm} \times 30 \mathrm{~cm} \times 20 \mathrm{~cm}$ y $70 \mathrm{~cm} \times 20 \mathrm{~cm} \times 20 \mathrm{~cm}$. Los tratamientos evaluados fueron: tres fermentaciones de biol en tiempos diferentes, dos coberturas inertes y dos densidades de siembra en el establecimiento y rendimiento del cultivo de Stevia. Se utilizó un diseño estadístico de Bloques Completos al Azar con arreglo trifactorial 3x2X2 +2 con cuatro repeticiones. Para el análisis de medias se realizó mediante la prueba de Tukey al 5\% de igual forma comparaciones ortogonales de los tratamientos en estudio. Las variables evaluadas fueron: Altura de la planta, diámetro del tallo, regeneración vegetativa, biomasa de malezas, rendimiento, concentración de nutrientes en las hojas y monitoreo de plagas y enfermedades. En cuanto a los resultados, los mejores rendimientos en producción de hoja seca, durante el ciclo productivo del cultivo fue el tratamiento T11 (Biol a los 75 días con Aserrín más densidad doble hilera) con 198.90 gramos hoja seca/10o pl en la primera cosecha, 644.10 gramos hoja seca/100 pl. en la segunda cosecha y, 1137.80 gramos hoja seca/10o pl en la tercera cosecha.

No hay presencia de interacciones entre los factores de estudio ( $a$, b y c) que influyan en el rendimiento de la hoja seca de Stevia. Las fermentaciones de biol inciden en el incremento productivo de materia seca. La fermentación de biol a los 75 días reporto los mejores valores con una producción de hoja seca de: $137.24 \mathrm{Kg} \mathrm{Hsha-1} \mathrm{(primera} \mathrm{cosecha),} 452.31 \mathrm{KgHsha-1} \mathrm{(segunda} \mathrm{cosecha)} \mathrm{y} 809.80 \mathrm{KgHsha-1}$ (tercera cosecha). Las densidades de siembra (factor c) no presentan diferencias significativas que gracias a su arquitectura y recuperación vegetal, permite altas densidades.

Palabras clave: Bioles, Stevia. hierba dulce, cultivo no tradicional 


\section{Abstract}

This research is aimed at evaluating the effect of different fermentations biol under inert cover systems on seeding in the establishment and crop yield nontraditional (Stevia rebaudiana). The research was conducted at the Experimental Farm of Agricultural Engineering Career Equinoctial Technological University, Campus Santo Domingo. 13,000 seedlings were planted Stevia, using two spacings of 70cm x 30cm x $20 \mathrm{~cm}$ and $70 \mathrm{~cm} \times 20 \mathrm{~cm} \times 20 \mathrm{~cm}$. The treatments were: three biol fermentations at different times, two coverages inert and two seeding in the establishment and yield of Stevia. We used a statistical design of randomized complete block under trifactorial $3 \times 2 \times 2+2$ with four replications. For the average analysis was performed using the Tukey test at 5\% Similarly orthogonal comparisons of the treatments under study. The variables evaluated were: plant height, stem diameter, vegetative regeneration, weed biomass, yield, nutrient concentration in leaves and pest and disease monitoring. As for the results, the best yields in dry leaf production during the crop production cycle was T11 treatment (Biol within 75 days with more density Sawdust double row) with 198.90 grams in leaf seca/100 pl first harvest, leaf seca/100 pl 644.10 grams. in the second harvest and 1137.80 grams pl seca/100 sheet in the third harvest.

No presence of interactions between the study factors ( $a, b)$ to influence the performance of Stevia dried leaf. Biol fermentations productive affect the increase of dry matter. Fermentation of biol at 75 days reported the highest production values with a dry leaf: 137.24 Kg HSHA-1 (first crop), 452.31 KgHsha-1 (second crop) and 809.80 KgHsha-1 ( third harvest). Planting densities (factor c) are not significantly different thanks to its architecture and vegetation recovery, allows high densities.

Keywords: Bioles, Stevia. sweet grass, traditional breeding 


\section{Introducción}

La Stevia tuvo una adaptación positiva en la zona de Santo Domingo y se hizo necesario conocer densidades de siembra que permitan obtener un alto rendimiento. Existen pocos trabajos que interrelacionen el desarrollo de cultivos bajo los efectos de una fertilización orgánica, sobre tipos de cobertura y diferentes densidades de siembra por lo que fue interesante su estudio.

Cabe señalar que se tiene experiencias de cultivos en alta densidad, con muy buenos resultados, plantando inclusive 200.000 plantas ha-1. Sin embargo, se deben observar algunos condicionantes para que esta práctica se lleve a cabo, que son: variedad mejorada (Morita II) por su arquitectura de planta permite alta densidad, riego complementario, cobertura de suelo, programa de fertilización y control de enfermedades fungosas (MAGP, 1996).

El empleo de biofertilizantes en los cultivos agrícolas es una alternativa para reducir la aplicación de fertilizantes químicos y de otros agroquímicos que dañan el medio ambiente, además que resultan 90\% más económicos para los agricultores nacionales. Con el uso de inoculantes biológicos (biofertilizantes), la incorporación de enmiendas orgánicas, las prácticas agrícolas que tienden a la conservación del suelo, la rotación de cultivos y el uso de leguminosas de cobertura, entre otras prácticas, pueden a largo plazo, contribuir a la recuperación de las poblaciones microbianas del suelo y con ello mejorar la calidad de este recurso (Cardoso et al., 1992; Siquiera \& Franco, 1998; Lindsey \& Jones, 1999).

Los resultados de innumerables estudios, junto con la toma de conciencia sobre los efectos adversos de los pesticidas químicos, propiciaron el resurgimiento a escala mundial de la investigación sobre el uso de inoculantes bacterianos para controlar patógenos y mejorar el crecimiento vegetal. De esta manera se utilizan organismos naturales (rizobacterias) para reducir los efectos de organismos indeseables (patógenos) y favorecer la producción de cultivos vegetales (Siquiera \& Franco, 1998; Perez et al., 2000).
También se pueden hacer abonos líquidos, con plantas cultivadas o silvestres, para lo cual se puede seleccionar dos o tres especies, procurando que una de ellas sea leguminosa, se pican bien las hierbas y ramas y se ponen en un recipiente con agua, en proporción de un kilogramo de la mezcla de hierbas picadas por cada dos litros de agua fresca, mantenga bien cerrado el recipiente y luego de tres o cuatro días, cierna bien y puede aplicar con una bomba de espalda o de motor, poniendo aproximadamente medio litro de esta solución, por bomba de 20 litros de agua, la cual puede aplicarse cada 30 a 60 días o más, dependiendo del estado del cultivo, se debe hacer especialmente en la época de floración y durante la maduración de los frutos (INIAP, 2001).

Las malezas son componentes naturales de un agro ecosistema y juegan un papel importante en la diversidad y estabilidad de las poblaciones de insectos. La mayor parte de los estudios sobre malezas se concentran en el control químico o cultural, pero generalmente no se mencionan los beneficios que éstas producen (ALTIERI, et al., 1977). Entre las prácticas culturales para el manejo de malezas están el uso de plantas de cobertura, el uso de coberturas muertas como plásticos, residuos de plantas, aserrín y cascarilla de arroz, así como variaciones de laboreo del suelo (Ramiro de la Cruz, 1997)

Según Savio y Zien, (2001), las cubiertas de materia orgánica inerte constituyen un buen método para el control de malezas y para mantener las plantas saludables. Una capa gruesa de mulching mantiene el suelo aislado, permite conservar la humedad (al reducir la evaporación), impide la erosión, evita que el follaje toque el suelo (lo que disminuye el riesgo de enfermedades de las plantas que se transmiten a través de éste) y mantiene las plagas alejadas.

El efecto físico es importante en la regulación de la germinación y emergencia de las plántulas en algunas especies, debido a que sus reservas no son suficientes para garantizar su sobrevivencia durante el espacio que deben recorrer a través de la "cubierta" hasta tener acceso a la luz (Pitelli , 1995). 


\section{Materiales y Métodos}

La presente investigación se desarrolló desde marzo del 2011 a enero del 2012 en la Granja Experimental de la Escuela de Ingeniería Agropecuaria en la Universidad Tecnológica Equinoccial, Campus Santo Domingo. Durante la investigación se evaluaron tres fermentaciones de biol en tiempos diferentes, dos coberturas inertes y dos densidades de siembra en el establecimiento y rendimiento del cultivo de Stevia. La descripción de los tratamientos se presenta en el Cuadro 1. Se utilizó un diseño de bloques completos al azar con arreglo trifactorial con cuatro repeticiones, dando un total de 56 unidades experimentales. Para la comparación de medias de los tratamientos se utilizó la prueba de Tukey al 5\% y comparaciones ortogonales de los tratamientos en estudio. Los parámetros evaluados fueron: altura de la planta, diámetro del tallo, regeneración vegetativa, biomasa de malezas, rendimiento, concentración de nutrientes en las hojas y monitoreo de plagas y enfermedades. La altura de planta se registró después del trasplante, midiéndolo desde el suelo hasta el punto terminal del ápice primario más alto, el diámetro de tallo se midió a $5 \mathrm{~cm}$ de altura desde el suelo, la regeneración vegetativa se procedió a contabilizar el número de brotes tiernos regenerados después de cada cosecha, la biomasa de las malezas se contabilizó mediante la técnica del metro cuadrado dando una relación del porcentaje, para el rendimiento se pesó la cantidad de hoja fresca y seca por tratamiento, la concentración de nutrientes en las hojas, para este parámetro se recolectó muestras de materia verde, se identificó y se envió al laboratorio para el análisis de macro y microelementos y para la incidencia de plagas y enfermedades, se efectuaron observaciones quincenales para identificar insectos y agentes fitopatógenos que afecten al cultivo de stevia.

\begin{tabular}{|c|c|c|}
\hline Simbología & Código & Tratamientos \\
\hline T1 & A1B1C1 & Biol a 45 días con C de Arroz a densidad 1 \\
T2 & A1B1C2 & Biol a 45 días con C de Arroz a densidad 2 \\
T3 & A1B2C1 & Biol a 45 días con A de Balsa a densidad 1 \\
T4 & A1B2C2 & Biol a 45 días con A de Balsa a densidad 2 \\
T5 & A2B1C1 & Biol a 60 días con C de Arroz a densidad 1 \\
T6 & A2B1C2 & Biol a 60 días con C de Arroz a densidad 2 \\
T7 & A2B2C1 & Biol a 60 días con A de Balsa a densidad 1 \\
T8 & A2B2C2 & Biol a 60 días con A de Balsa a densidad 2 \\
T9 & A3B1C1 & Biol a 75 días con C de Arroz a densidad 1 \\
T10 & A3B1C2 & Biol a 75 días con C de Arroz a densidad 2 \\
T11 & A3B2C1 & Biol a 75 días con A de Balsa a densidad 1 \\
T12 & A3B2C2 & Biol a 75 días con A de Balsa a densidad 2 \\
T13 & To & Testigo absoluto a densidad 1 \\
T14 & Ti & Testigo absoluto a densidad 2 \\
\hline
\end{tabular}

Cuadro 1.

Descripción de los tratamientos evaluados en la investigación.

\section{Discusión y resultados}

\section{Altura de planta de Stevia rebaudiana $(\mathrm{cm}$.)}

En el cuadro 2 se observa los resultados de los análisis de varianza para la altura de la planta durante las tres cosechas. Se observa alta significancia estadística entre los tratamientos y para la comparación entre factores (Fermentación de biol, coberturas y densidades de siembra) vs adicionales (testigos), evaluados durante los tres cortes productivos de Stevia rebaudiana. Se mantiene una significativa interacción entre las fermentaciones de biol con las distintas densidades de siembra que se evaluaron en el campo durante la primera cosecha. En la segunda cosecha del cultivo se presentó una alta significancia para la densidades de siembra (Factor b) para esta variable. Por lo tanto existe influencia del uso de biol a diferentes densidades de siembra, sobre la variable en mención, confirmando la hipótesis de que las diferentes fermentaciones de biol con diferentes coberturas sobre densidades de siembra ejercen una influencia positiva sobre la altura del cultivo. Los coeficientes de variación fueron: $8,14 \%, 10,92 \%$ y 13,51 \%, que son aceptables. 


\begin{tabular}{|c|c|c|c|c|c|c|c|}
\hline F.V. & $\mathrm{gl}$ & \multicolumn{2}{|c|}{ Primera cosecha } & \multicolumn{2}{|c|}{ Segunda cosecha } & \multicolumn{2}{|c|}{ Tercera cosecha } \\
\hline Total & 55 & & & & & & \\
\hline Tratamientos & 13 & 4.26 & $* *$ & 5.42 & ** & 5.37 & ** \\
\hline Bioles (a) & 2 & 0.52 & $\mathrm{~ns}$ & 0.45 & ns & 0.95 & ns \\
\hline Coberturas (b) & 1 & 1.63 & $\mathrm{~ns}$ & 0.52 & ns & 2.44 & ns \\
\hline$a * b$ & 2 & 0.10 & ns & 0.11 & ns & 1.00 & ns \\
\hline Densidades ${ }^{(C)}$ & 1 & 2.84 & ns & 6.94 & * & 3.06 & ns \\
\hline$a^{*} c$ & 2 & 3.45 & * & 0.67 & ns & 0.36 & ns \\
\hline$b^{*} \mathrm{c}$ & 1 & 0.20 & ns & 0.08 & ns & 0.70 & ns \\
\hline$a^{*} b^{*} c$ & 2 & 1.08 & ns & 0.56 & ns & 0.04 & ns \\
\hline Factores vs Adicionales & 1 & 40.23 & ** & 58.58 & ** & 58.94 & ** \\
\hline Testigo o vs Ti & 1 & 0.15 & ns & 0.82 & ns & 0.01 & ns \\
\hline Repeticiones & 3 & 14.15 & & 12.65 & & 6.89 & \\
\hline Error & 39 & & & & & & \\
\hline \multicolumn{2}{|c|}{ Coeficiente de Variación (\%): } & 8.14 & & 10.92 & & 13.51 & \\
\hline
\end{tabular}

Cuadro 2. Análisis de varianza para altura de planta de Stevia rebaudiana, durante tres cortes productivos.

Según la prueba de Tukey al $5 \%$, en el análisis de los tratamientos en el primer corte productivo de Stevia rebaudiana, presentan tres rangos de significancia. En el primer rango se ubicaron: T11 (Biol a los 75 días con cobertura de Aserrín más densidad doble hilera) con una altura de $40.33 \mathrm{~cm}$, T8 (Biol a los 60 días con cobertura de aserrín más densidad triple hilera) con $40.05 \mathrm{~cm}$ y T4 (Biol a los 45 días con cobertura de aserrín más densidad triple hilera) con un promedio de $39,38 \mathrm{~cm}$ son estadísticamente iguales y superiores al resto de tratamientos. En el segundo corte productivo el ciclo de crecimiento de la planta se acelera y esto se comprueba en la prueba de Tukey que reporta que los mejores tratamientos son: T2 (Biol a 45 días con cáscara de Arroz a densidad triple hilera) con 39.35 cm,T10 (Biol a los 75 días con cáscara de Arroz más densidad triple hilera) $38.48 \mathrm{~cm}$, T12 (Biol a los 75 días con cobertura de aserrín más densidad triple hilera) 38.18 cm, T8 (Biol a los 60 días con aserrín más densidad triple hilera) con $38.00 \mathrm{~cm}$, T4 (Biol a los 45 días con cobertura de aserrín más densidad triple hilera) $37.38 \mathrm{~cm}$, T5 (Biol a los 60 días con cáscara de arroz más densidad doble hilera) con $37.20 \mathrm{~cm}$ y T6 (Biol a los 60 días con cáscara de arroz más densidad triple hilera) con una media de $37.20 \mathrm{~cm}$, siendo diferentes y superiores al resto de tratamientos evaluados.

En el tercer corte productivo de Stevia rebaudiana, se reportó dos rangos de significancia. En el primer rango se encuentran: T10, T2, T9, T6, T12, T8, T11, T7, T4 y T1; que son estadísticamente iguales y superiores al resto de tratamientos con promedios de altura que van de $40.40 \mathrm{~cm}$ a $34.68 \mathrm{~cm}$. Estos resultados se observan en el Cuadro 3, que se aprecia la evolución de la altura de planta en los diferentes cortes productivos que probaron en este ensayo.

Basantes.2009, menciona quelafunción delbiolprincipalmente al interior de las plantas, activando el fortalecimiento del equilibrio nutricional como un mecanismo de defensa de las mismas, a través de las hormonas de crecimiento, antibióticos, vitaminas, minerales, enzimas y coenzimas, carbohidratos, aminoácidos y azucares complejas, entre otros, presentes en la complejidad de las relaciones biológicas, químicas, físicas e energéticas que se establecen entre las plantas y la vida del suelo.

\begin{tabular}{|c|c|c|c|c|c|c|c|c|c|}
\hline \multicolumn{4}{|c|}{ Tratamientos } & \multicolumn{6}{|c|}{ Altura de planta (cm.) } \\
\hline \# & Biol & Cobertura & Densidad & Primera cc & echa & Segunda cc & & Tercera co & \\
\hline 1 & 45 días & C. de arroz & doble & 35.23 & $a b c$ & 33.85 & $a b c$ & 34.68 & $\mathrm{a}$ \\
\hline 2 & 45 días & C. de arroz & triple & 37.60 & $a b$ & 39.35 & $\mathrm{a}$ & 40.15 & $\mathrm{a}$ \\
\hline 3 & 45 días & Aserrín & doble & 35.83 & $a b c$ & 33.43 & $a b c$ & 32.68 & $\mathrm{ab}$ \\
\hline 4 & 45 días & Aserrín & triple & 39.38 & $\mathrm{a}$ & 37.38 & a & 35.03 & $\mathrm{a}$ \\
\hline 5 & 60 días & C. de arroz & doble & 35.43 & $a b c$ & 37.20 & $\mathrm{a}$ & 33.98 & $\mathrm{ab}$ \\
\hline 6 & 60 días & C. de arroz & triple & 38.10 & $a b$ & 37.20 & $\mathrm{a}$ & 36.05 & $\mathrm{a}$ \\
\hline 7 & 60 días & Aserrín & doble & 36.50 & $a b c$ & 34.15 & $a b$ & 35.20 & $\mathrm{a}$ \\
\hline 8 & 60 días & Aserrín & triple & 40.05 & $\mathrm{a}$ & 38.00 & $\mathrm{a}$ & 35.98 & $\mathrm{a}$ \\
\hline 9 & 70 días & C. de arroz & doble & 37.58 & $a b$ & 36.15 & $\mathrm{ab}$ & 37.63 & $\mathrm{a}$ \\
\hline 10 & 70 días & C. de arroz & triple & 38.00 & $a b$ & 38.48 & $\mathrm{a}$ & 40.40 & $\mathrm{a}$ \\
\hline 11 & 70 días & Aserrín & doble & 40.33 & $\mathrm{a}$ & 36.33 & $a b$ & 35.50 & $\mathrm{a}$ \\
\hline 12 & 70 días & Aserrín & triple & 36.43 & $a b c$ & 38.18 & $\mathrm{a}$ & 36.03 & a \\
\hline 13 & Testigo 1 & & doble & 29.93 & $\mathrm{c}$ & 26.68 & $\mathrm{bc}$ & 22.53 & b \\
\hline 14 & Testigo 2 & & triple & 30.75 & $\mathrm{bc}$ & 24.23 & $\mathrm{c}$ & 22.63 & b \\
\hline
\end{tabular}

Cuadro 3. Análisis de Tukey al 5\% para la variable altura de planta Stevia rebaudiana, durante tres cortes productivos.

En la Figura 1, se observan la interacción entre los Bioles (factor a) y las densidades de siembra (factor $\mathrm{c}$ ) que se probaron en este ensayo y su incidencia en la primera cosecha productiva. En el lapso de tiempo posterior a las siguientes cosechas la interacción se reportó imperceptible en la altura de planta de Stevia rebaudiana. La densidad de siembra a doble hilera, reportó una influencia lineal a los 75 días de fermentación del biol, la altura de planta se incrementa con un promedio de $38.95 \mathrm{~cm}$, a diferencia de la densidad triple hilera que se obtuvo mayor respuesta con el biol fermentado durante 45 días con $38.74 \mathrm{~cm}$ y con el biol fermentado durante 60 días con una altura de planta de $39.08 \mathrm{~cm}$, además se observa un decrecimiento de la planta con la densidad doble hilera, usando el biol fermentado durante 75 días. 


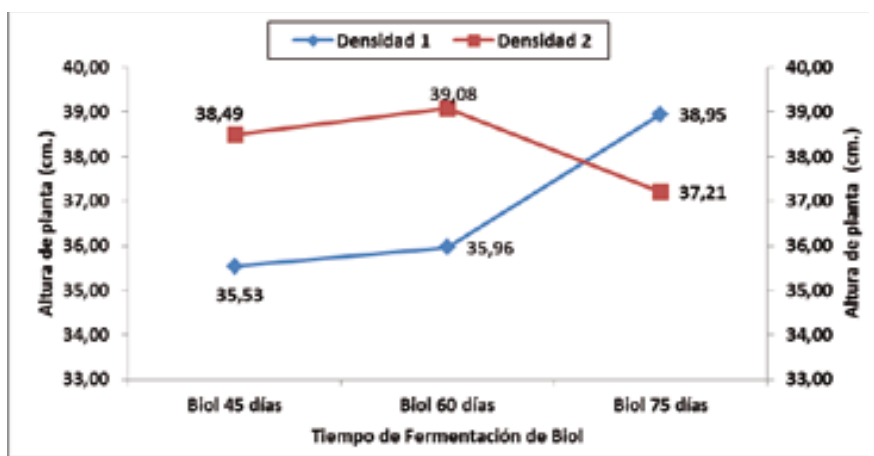

Figura 1. Influencia de las fermentaciones de Biol en las densidades de siembra en el primer corte productivo de Stevia rebaudiana.

En la Figura 2 se observa el comportamiento de la altura de la planta durante las tres cosechas con dos densidades de siembra. La densidad de siembra (factor c) presenta significancia estadística en el segundo corte productivo y según la prueba de Tukey al $5 \%$, la densidad con triple hilera muestra un promedio de altura de planta de $38.10 \mathrm{~cm}$ siendo superior a la densidad con doble hilera, con una altura de planta de $35.18 \mathrm{~cm}$. Según los resultados obtenidos se indica la relevancia de la densidad con doble hilera, en altura de planta en las diferentes etapas del cultivo de Stevia rebaudiana, con un notorio incremento en la segunda y tercera cosecha.

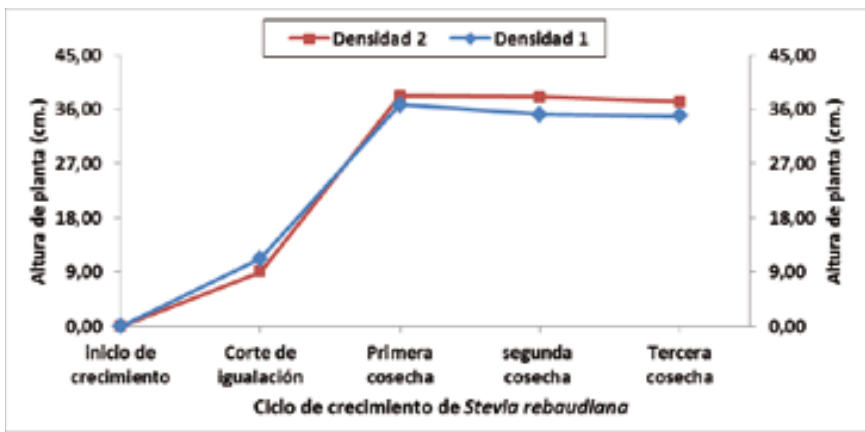

Figura 2. Comportamiento de la altura de planta durante tres cosechas de Stevia a dos densidades de siembra.

La densidad de siembra y los rendimientos óptimos fluctúan de una zona agro ecológica a otra. De acuerdo a lo citado por Amaya, 2010 quien señala que la densidad de siembra de $0.25 \times 0.40 \mathrm{~m}$, alcanzó los mejores resultados para altura de planta en la Stevia, con un promedio de $26.16 \mathrm{~cm}$.

El hábito de crecimiento de la planta su morfología y condiciones ambientales influyen en la altura de planta, obteniendo en este ensayo, con una densidad de $0.20 \times 0.30 \mathrm{~m}$, una altura promedio de $36.81 \mathrm{~cm}$ y con la densidad $0.20 \times 0.20 \mathrm{~m}$, un valor promedio de $38.08 \mathrm{~cm}$, siendo superiores a los valores reportados por Amaya, 2010.

\section{Diámetro de tallo en Stevia rebaudiana}

Los datos del análisis de varianza del diámetro del tallo se presentan en el Cuadro 4, donde reportan diferencias significativas en la primera cosecha y alta significancia durante el segundo y tercera corte. En el segundo corte productivo presente significancia estadística en los tiempos de fermentación del biol (factor a) y en la interacción entre los tiempos de fermentación del biol, las coberturas inertes (factor b) y las densidades de siembra (factor c). Los coeficientes de variación fueron: $23.40 \%, 8.52 \%$ y $13.51 \%$ respectivamente.

\begin{tabular}{|c|c|c|c|c|c|c|c|}
\hline F.V. & $\mathrm{gl}$ & Primera cosec & & Segunda co & echa & Tercera co & echa \\
\hline Total & 55 & & & & & & \\
\hline Tratamientos & 13 & 2.00 & $*$ & 11.15 & $* *$ & 2.96 & $* *$ \\
\hline Bioles (a) & 2 & 1.50 & ns & 3.57 & $*$ & 1.50 & ns \\
\hline Coberturas (b) & 1 & 1.00 & $\mathrm{~ns}$ & 3.57 & $\mathrm{~ns}$ & 4.00 & ns \\
\hline $\mathrm{a}{ }^{*} \mathrm{~b}$ & 2 & 1.00 & $\mathrm{~ns}$ & 0.07 & $\mathrm{~ns}$ & 0.50 & ns \\
\hline Densidades $\mathbb{C}$ & 1 & 1.50 & $\mathrm{~ns}$ & 0.96 & $\mathrm{~ns}$ & 1.00 & ns \\
\hline$a^{*} c$ & 2 & 1.00 & $\mathrm{~ns}$ & 0.20 & ns & 0.50 & ns \\
\hline$b^{*} \mathrm{c}$ & 1 & 1.50 & ns & 0.30 & $\mathrm{~ns}$ & 1.50 & ns \\
\hline$a * b * c$ & 2 & 1.50 & $\mathrm{~ns}$ & 3.57 & $*$ & 0.50 & $\mathrm{~ns}$ \\
\hline Factores vs Adicionales & 1 & 8.90 & $* *$ & 129.34 & $* *$ & 26.94 & $* *$ \\
\hline Testigo o vs $\mathrm{Ti}$ & 1 & 0.04 & $\mathrm{~ns}$ & 1.60 & ns & 0.02 & ns \\
\hline Repeticiones & 3 & 2.20 & $\mathrm{~ns}$ & 1.74 & ns & 1.46 & ns \\
\hline Error & 39 & & & & & & \\
\hline Coeficiente de Variación & $(\%):$ & 23.40 & & 8.52 & & 13.51 & \\
\hline
\end{tabular}

Cuadro 4. Análisis de varianza para el diámetro de tallo de Stevia rebaudiana, durante tres cortes productivos.

El uso de diferentes fermentaciones de biol, en función con las coberturas inertes y las diferentes densidades de siembra en Stevia rebaudiana, inciden de forma favorable en el diámetro del tallo de las plantas, que se evaluaron en esta investigación. De acuerdo a la 
comparación estadística de estos factores vs adicionales (Testigos), presentó alta significancia, durante el ciclo de cultivo. Por lo tanto existe influencia positiva de los factores en estudio sobre el cultivo de la Stevia.

En el Cuadro 5 se observa el análisis de la prueba de Tukey al 5\% de probabilidad, para diámetro de la planta. Según los datos, durante el primer corte productivo de Stevia, reporta dos rangos significativos, en el primer rango se ubica T5 (biol a los 60 días con C de Arroz mas densidad doble hilera) con $0.82 \mathrm{~cm}$. Los promedios más bajos se presentaron en los testigos de la investigación (T13, T14) con $0.44 \mathrm{~cm}$ y $0.42 \mathrm{~cm}$, en grosor de tallo respectivamente. En el segundo corte productivo los testigos T13 y T14 se mantienen inferiores al resto de tratamientos, con promedios que están entre $0.45 \mathrm{~cm}$ y $0.40 \mathrm{~cm}$. En el tercer corte productivo, reportan valores superiores al resto de cortes: T5 (biol a los 60 días con C de Arroz mas densidad doble hilera) con un diámetro de $0.92 \mathrm{~cm}$ y $\mathrm{T} 1$ (biol a los 45 días con cáscara de arroz mas densidad doble hilera) con $0.90 \mathrm{~cm}$ en promedio. Los tratamientos T13 (testigo doble hilera) de $0.50 \mathrm{~cm}$ y T14 (testigo triple hilera) con 0.51 $\mathrm{cm}$, son estadísticamente inferiores al resto de tratamientos.

Rivero, 1999, argumenta que el uso de bioles, promueve las actividades fisiológicas y estimula el desarrollo de las plantas, sirviendo para las siguientes actividades agronómicas; acción sobre el follaje, floración, cuajado de frutos, enraizamiento y activador de semillas y partes vegetativas.

\begin{tabular}{|c|c|c|c|c|c|c|c|c|c|}
\hline \multicolumn{4}{|c|}{ Tratamientos } & \multicolumn{6}{|c|}{ Diámetro de tallo $(\mathrm{cm})}$. \\
\hline \# & Biol & Cobertura & Densidad & Primera co & & Segunda cosecha & & Tercera cos & \\
\hline 1 & 45 días & C. de arroz & doble & 0.55 & $\mathrm{ab}$ & 0.72 & $\mathrm{a}$ & 0.90 & a \\
\hline 2 & 45 días & C. de arroz & triple & 0.51 & $a b$ & 0.68 & $\mathrm{a}$ & 0.85 & $a b$ \\
\hline 3 & 45 días & Aserrín & doble & 0.54 & $a b$ & 0.67 & $\mathrm{a}$ & 0.80 & $a b$ \\
\hline 4 & 45 días & Aserrín & triple & 0.57 & $a b$ & 0.66 & $\mathrm{a}$ & 0.75 & $a b$ \\
\hline 5 & 60 días & C. de arroz & doble & 0.82 & $\mathrm{a}$ & 0.64 & $\mathrm{a}$ & 0.92 & a \\
\hline 6 & 60 días & C. de arroz & triple & 0.55 & $a b$ & 0.67 & $\mathrm{a}$ & 0.80 & $a b$ \\
\hline 7 & 60 días & Aserrín & doble & 0.55 & $a b$ & 0.66 & $\mathrm{a}$ & 0.76 & $a b$ \\
\hline 8 & 60 días & Aserrín & triple & 0.57 & $\mathrm{ab}$ & 0.62 & $\mathrm{a}$ & 0.73 & $a b$ \\
\hline 9 & 75 días & C. de arroz & doble & 0.58 & $a b$ & 0.68 & $\mathrm{a}$ & 0.79 & $a b$ \\
\hline 10 & 75 días & C. de arroz & triple & 0.57 & $a b$ & 0.63 & $\mathrm{a}$ & 0.72 & $a b$ \\
\hline 11 & 75 días & Aserrín & doble & 0.58 & $a b$ & 0.62 & $\mathrm{a}$ & 0.66 & $a b$ \\
\hline 12 & 75 días & Aserrín & triple & 0.51 & $a b$ & 0.64 & $\mathrm{a}$ & 0.78 & $a b$ \\
\hline 13 & Testigo 1 & & doble & 0.44 & $\mathrm{~b}$ & 0.45 & b & 0.50 & b \\
\hline 14 & Testigo 2 & & triple & 0.42 & $\mathrm{~b}$ & 0.40 & b & 0.51 & b \\
\hline
\end{tabular}

Cuadro 5. Análisis de Tukey al 5\% para la variable diámetro de planta Stevia rebaudiana, durante tres cortes productivos.
En la Figura 3, se observa la interacción que influye en el diámetro de tallo de la Stevia rebaudiana, entre los tiempos fermentaciones de biol (factor a) y las densidades de siembra (factor c) evaluados en el segundo corte productivo. La fermentación de biol a los 45 días influye significativamente en la amplitud del tallo en las diferentes densidades de siembra con valores promedios de 0.70 $\mathrm{cm}$ en la densidad doble hilera y $0.67 \mathrm{~cm}$ en la densidad triple hilera. Además se observa un menor efecto en la fermentación de biol a los 75 días con la densidad de siembra triple hilera, con una media de 0.64 $\mathrm{cm}$ en grosor de tallo.

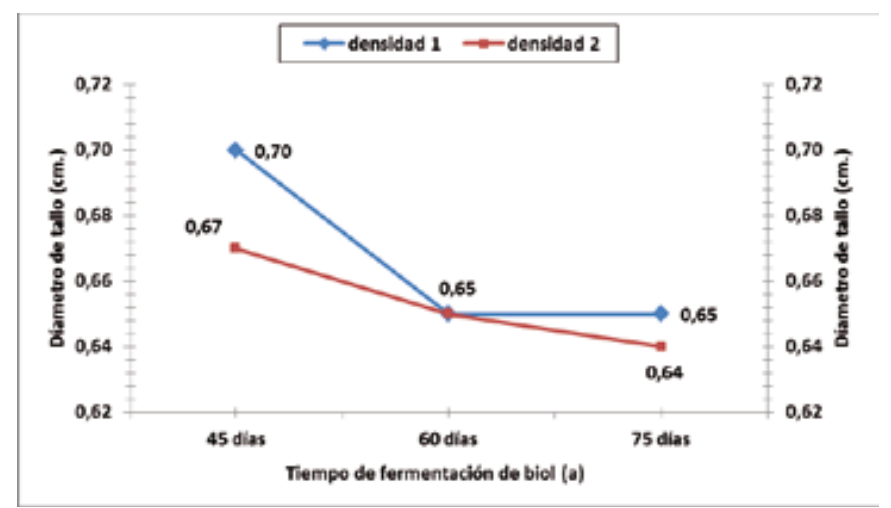

Figura 3. Influencia de las fermentaciones de Biol en las densidades de siembra en el segundo corte productivo de Stevia rebaudiana.

En la Figura 4, se observa la interacción de las coberturas inertes (factor b) que inciden en el diámetro del tallo, con las diferentes densidades de siembra, evaluados en la segunda cosecha productiva de Stevia rebaudiana. El uso de la cobertura de cascarilla de arroz presenta los mejores valores en diámetro de tallo con la densidad doble hilera, con un promedio de $0.68 \mathrm{~cm}$ y $0.66 \mathrm{~cm}$ en la densidad triple hilera; esto resultados difieren con los valores presentes en la cobertura a base de aserrín de madera que fueron: $0.65 \mathrm{~cm}$ y $0.64 \mathrm{~cm}$ respectivamente. 


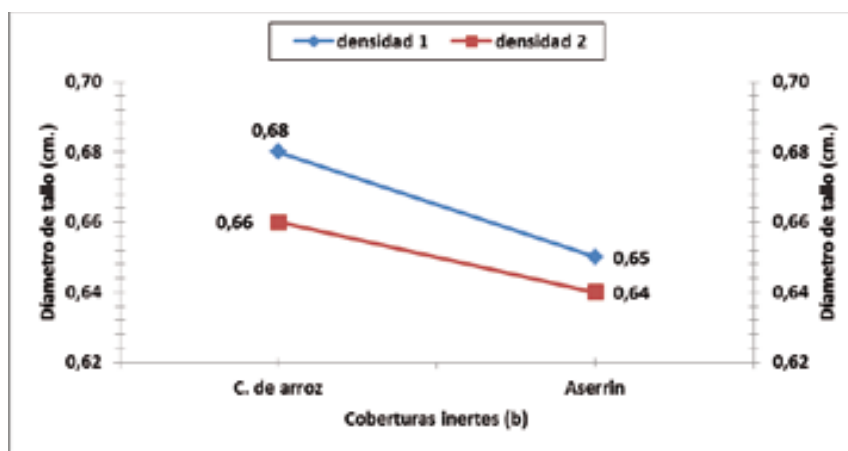

Figura 4. Influencia de las Cobertura inertes en las densidades de siembra en el segundo corte productivo de Stevia rebaudiana.

Según Birchler 1998, indica que el diámetro del tallo, está influenciado por la densidad del cultivo y puede verse afectado por prácticas culturales. Siendo el diámetro un indicador de la densidad de cultivo.

Ortiz, 2004 manifiesta que "El acolchado o "mulching" protege la estructura superficial del suelo frente al impacto de la gota de agua, evita los cambios bruscos de temperatura entre el día y la noche, mejora la tasa de infiltración, las pérdidas por escorrentía y la erosión, y reduce el crecimiento de las malas hierbas, esto genera un mayor crecimiento radicular, especialmente en plantas jóvenes y en áreas de veranos muy cálidos".

\section{Recuperación Vegetativa (brotes/planta)}

En el Cuadro 6, presenta el análisis de varianza para esta variable, evaluados durante tres cortes productivos de la Stevia rebaudiana. Para coberturas inertes (Factor b), reporta significancia estadística en las coberturas inertes (Factor b) en el primer corte productivo y en los tratamientos en el segundo corte. En el tercer corte productivo hay alta significancia estadística en los tratamientos, fermentaciones de biol (Factor a), coberturas inertes (Factor b) y significancia en las densidades de siembra (Factor C). Los coeficientes de variación en esta variable fueron: $15.40 \%$, $24.42 \%$ y $6.37 \%$.

\begin{tabular}{|c|c|c|c|c|c|c|c|}
\hline F.V. & $\mathrm{gl}$ & Primera cosecha & & nda cosecha & & Tercera cos & \\
\hline Total & 55 & & & & & & \\
\hline Tratamientos & 13 & 0.99 & ns & 2.28 & * & 16.33 & ** \\
\hline Bioles (a) & 2 & 0.43 & ns & 0.77 & ns & 15.05 & ** \\
\hline Coberturas (b) & 1 & 4.61 & * & 0.52 & $\mathrm{~ns}$ & 9.30 & ** \\
\hline$a * b$ & 2 & 0.97 & ns & 0.55 & ns & 7.72 & ** \\
\hline Densidades $\mathbb{C}$ & 1 & 0.09 & ns & 0.00 & ns & 4.22 & * \\
\hline$a^{*} c$ & 2 & 0.35 & ns & 1.12 & ns & 3.36 & * \\
\hline$b^{*} \mathrm{c}$ & 1 & 1.36 & $\mathrm{~ns}$ & 0.25 & ns & 0.12 & $\mathrm{~ns}$ \\
\hline$a^{*} b^{*} c$ & 2 & 0.22 & ns & 2.03 & ns & 0.32 & $\mathrm{~ns}$ \\
\hline Factores vs Adicionales & 1 & 1.44 & ns & 18.36 & ** & 142.41 & ** \\
\hline Testigo o vs Ti & 1 & 1.38 & ns & 1.61 & ns & 3.33 & $\mathrm{~ns}$ \\
\hline Repeticiones & 3 & 6.07 & & 15.22 & & 12.33 & \\
\hline Error & 39 & & & & & & \\
\hline Coeficiente de Variación & $(\%):$ & 15.40 & & 24.42 & & 6.37 & \\
\hline
\end{tabular}

Cuadro 6. Análisis de varianza para recuperación vegetativa en Stevia rebaudiana, durante tres cortes productivos.

De acuerdo al análisis estadístico hay presencia de interacciones en el tercer corte productivo del cultivo, además se observa alta significancia estadística en la interacción a*b (F. de biol * coberturas inertes) y significancia estadística entre $a^{*} \mathrm{c}$ (F. de biol * densidades de siembra). Los factores (F de biol, coberturas inertes y densidades de siembra) en comparación con los Adicionales (Testigos control) no presenta diferencia significativas en el primer corte productivo. A diferencia de los dos últimos cortes que se reporta altamente significativo, lo que demuestra la influencia de los factores en estudio en la recuperación vegetativa de la Stevia rebaudiana.

En el Cuadro 7 se observa el análisis de la prueba de Tukey al 5\% de probabilidad, para la variable recuperación vegetativa. Según los resultados obtenidos durante el primer corte productivo del cultivo, no presentó diferencias significativas, la proliferación de brotes se produce en acción de la fisiología de la planta. En el segundo corte productivo, se detectaron dos rangos de significancia, en el primer rango se ubicaron T2 (Biol a los 45 días con C de arroz más densidad triple hilera) con un promedio de 28.28 brotes/ planta y T11 (Biol a los 45 días con C de arroz más densidad triple hilera) con 27.58 brotes/ planta; siendo estadísticamente iguales y superiores al resto. Con los valores más bajo tenemos al T14 (Testigo triple hilera) con una proliferación de 12.33 brotes/planta. 
Durante el desarrollo para el tercer corte productivo, presentaron según Tukey $5 \%$, tres rangos significativos. Los tratamientos $\mathrm{T} 13$ (Testigo doble hilera) con 93.05 brotes/ planta y T14 (Testigo triple hilera)con 83.40 brotes/planta; siendo estadísticamente iguales y ocupando el último rango. En tanto que los T3, T4, T5, T6, T7, T8, T9, T10, T11 y T12 son estadísticamente iguales y superiores al resto de tratamientos con valores promedios entre 129.65 brotes/planta y 119.38 brotes/planta.

Guevara, 2011 indica que "El biol promueve las actividades fisiológicas y estimula el desarrollo de las plantas, acción sobre la floración, incidencia en el follaje y enraizamiento. El 92\% de la cosecha depende de la actividad fotosintética y el $8 \%$ de los nutrimentos que la planta extrae del suelo". Los valores promedios en proliferación de brotes de Stevia que se observan en el Cuadro 7, coincide con lo expuesto por Guevara quien señala que las aplicaciones de biol influyen en la respuesta fisiológica de la planta en su recuperación vegetativa.

\begin{tabular}{|c|c|c|c|c|c|c|c|c|c|}
\hline \multicolumn{4}{|c|}{ Tratamientos } & \multicolumn{6}{|c|}{ Recuperación vegetativa (\# de brotes/planta) } \\
\hline \# & Biol & Cobertura & Densidad & Primera & osecha & Segunda $\mathrm{c}$ & & Tercera co & \\
\hline 1 & 45 días & C. de arroz & doble & 7.98 & $\mathrm{a}$ & 23.35 & $\mathrm{ab}$ & 97.78 & $\mathrm{bc}$ \\
\hline 2 & 45 días & C. de arroz & triple & 8.05 & $\mathrm{a}$ & 28.28 & a & 112.98 & $\mathrm{ab}$ \\
\hline 3 & 45 días & Aserrín & doble & 8.38 & $\mathrm{a}$ & 24.95 & ab & 119.38 & a \\
\hline 4 & 45 días & Aserrín & triple & 7.50 & a & 22.15 & $a b$ & 128.43 & a \\
\hline 5 & 60 días & C. de arroz & doble & 8.90 & $\mathrm{a}$ & 24.33 & $a b$ & 121.85 & a \\
\hline 6 & 60 días & C. de arroz & triple & 8.78 & a & 22.85 & $\mathrm{ab}$ & 123.00 & a \\
\hline 7 & 60 días & Aserrín & doble & 8.00 & $\mathrm{a}$ & 18.35 & ab & 122.43 & $\mathrm{a}$ \\
\hline 8 & 60 días & Aserrín & triple & 7.68 & a & 24.10 & ab & 125.65 & $\mathrm{a}$ \\
\hline 9 & 75 días & C. de arroz & doble & 8.13 & $\mathrm{a}$ & 24.25 & $a b$ & 129.65 & $\mathrm{a}$ \\
\hline 10 & 75 días & C. de arroz & triple & 9.10 & $\mathrm{a}$ & 23.03 & ab & 128.85 & $\mathrm{a}$ \\
\hline 11 & 75 días & Aserrín & doble & 7.58 & $\mathrm{a}$ & 27.58 & a & 129.48 & $\mathrm{a}$ \\
\hline 12 & 75 días & Aserrín & triple & 7.20 & $\mathrm{a}$ & 22.1 & $a b$ & 128.25 & $\mathrm{a}$ \\
\hline 13 & Testigo 1 & & doble & 8.05 & $\mathrm{a}$ & 17.25 & $a b$ & 93.05 & $\mathrm{c}$ \\
\hline 14 & Testigo 2 & & triple & 7.03 & $\mathrm{a}$ & 12.33 & b & 83.4 & $\mathrm{c}$ \\
\hline
\end{tabular}

Cuadro 7. Análisis de Tukey al 5\% para la variable recuperación vegetativa Stevia rebaudiana, durante tres cortes productivos.

En la Figura 5, se observa la interacción entre las fermentaciones de biol (Factor a) y las coberturas inertes (Factor b) presente en el tercer corte productivo de Stevia rebaudiana. La influencia de estos factores en la proliferación de brotes favorece el rendimiento del cultivo, el uso de cobertura de cascarilla de arroz (b1) en este ensayo al interactuar con la fermentación de biol a los 45 días (a1) presento una baja emisión en promedio de 105.38 brote/ planta, al aplicar biol fermentado durante 60 días (a2) y 75 días (a3) la recuperación vegetativa, presento un comportamiento lineal positivo con valores de 12243 brote/planta a 129.25 brotes/planta. La diferencia en la cobertura a base de aserrín de madera (b2) y la aplicación de biol de 45 días de fermentación presento mayor respuesta que en la cascarilla de arroz (b1) con un promedio de 123.90 brotes/planta.

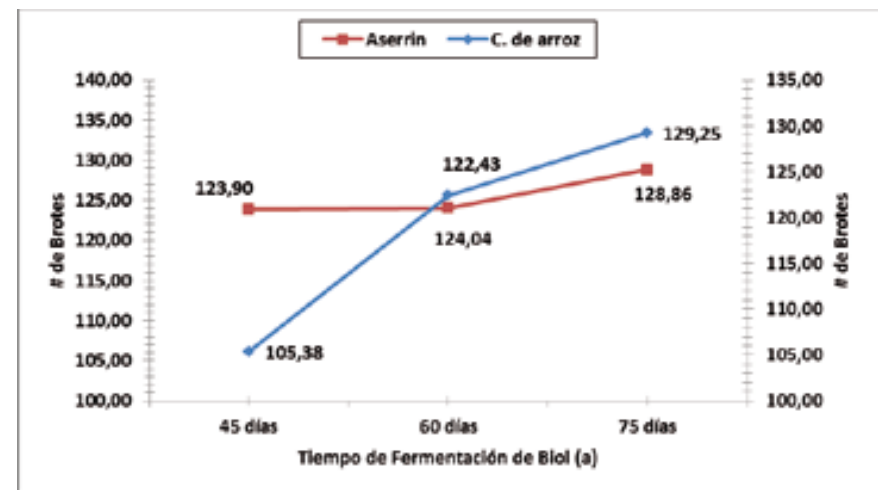

Figura 5. Influencia de las fermentaciones de Biol y las cobertura inertes en la recuperación vegetativa en el tercer corte productivo de Stevia rebaudiana.

Ortiz, 2004 argumenta: "Las coberturas tienen efecto biológico y se debería a que se crean las condiciones para el desarrollo de una importante y diversificada población de microorganismos, los que ejercen funciones de deterioro y pérdida de viabilidad de diversos propágulos en el suelo, y también genera un ambiente seguro para predadores de semillas y plántulas".

En la Figura 6, se observa la influencia de los tiempos de fermentación del biol (factor a), y las densidades de siembra (b) sobre la recuperación vegetativa durante el tercer corte. La influencia de estos factores es significativo en la emisión de brotes de Stevia rebaudiana. Las fermentaciones de biol a los 45 días (a1) y 60 días (a2) reportan mayores efectos en la densidad triple hilera, con promedios de recuperación vegetativa de 120.70 brotes/planta a 124.33 brotes/ planta, en comparación con la densidad doble hilera, con valores de 108.58 brotes/planta y 122.14 brotes/planta. En la aplicación de biol fermentado a los 75 días (a3) la densidad doble hilera, reporta mayor incidencia con una formación de 129.56 brotes/planta, siendo superior

\section{Tsafiquilewax}


a la densidad triple hilera, con una recuperación vegetativa de 128.55 brotes/planta comparativamente.

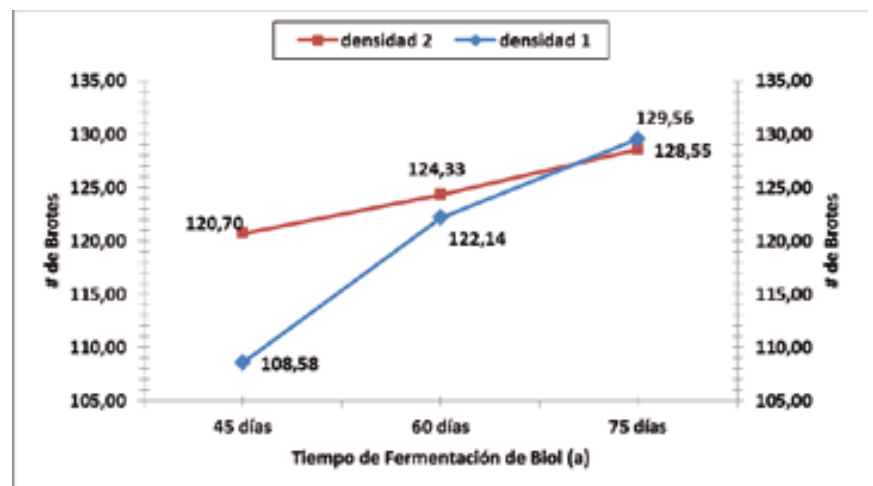

Figura 6. Influencia de las fermentaciones de Biol en las densidades de siembra, para la recuperación vegetativa durante el tercer corte productivo de Stevia rebaudiana.

Alcivar y Parraga, 2012, indican que "Las aplicaciones de biol al suelo y al follaje, no solo mejoran la estructura, sino también por los contenidos de hormonas y precursores favorece el desarrollo de las plantas sin que el funcionamiento de su metabolismo sea alterado y una mayor actividad de microorganismos del suelo".

\section{Rendimiento en hoja de Stevia rebaudiana}

En el Cuadro 8 se revela el análisis de varianza del rendimiento en hoja seca durante tres cosechas productivas de Stevia rebaudiana. Para la primera cosecha de hoja no se obtuvo ninguna significancia estadística. A partir de la segunda y tercera cosecha, presentó alta significancia estadística entre los tratamientos en estudio y significancia en las fermentaciones de biol (Factor a) aislado en el segundo corte productivo. Los coeficientes de variación se mantuvieron en: $20.09 \%$, $23.46 \%$ y $25.38 \%$; que es aceptable en la investigación.

\begin{tabular}{|c|c|c|c|c|c|c|c|}
\hline F.V. & $\mathrm{gl}$ & \multicolumn{2}{|c|}{ Primera cosecha } & \multicolumn{2}{|l|}{ Segunda cosecha } & \multicolumn{2}{|l|}{ Tercera cosecha } \\
\hline Total & 55 & & & & & & \\
\hline Tratamientos & 13 & 0.64 & ns & 2.72 & $* *$ & 4.43 & ** \\
\hline Bioles (a) & 2 & 0.74 & ns & 3.26 & $*$ & 0.34 & ns \\
\hline Coberturas (b) & 1 & 0.18 & ns & 2.40 & ns & 0.01 & ns \\
\hline $\mathrm{a} * \mathrm{~b}$ & 2 & 0.73 & ns & 0.55 & ns & 1.20 & $\mathrm{~ns}$ \\
\hline Densidades ${ }^{C}$ & 1 & 1.08 & ns & 2.84 & ns & 1.32 & ns \\
\hline$a^{*} c$ & 2 & 0.82 & ns & 0.46 & ns & 0.43 & ns \\
\hline$b^{*} \mathrm{c}$ & 1 & 0.11 & ns & 2.07 & ns & 0.07 & ns \\
\hline$a^{*} b^{*} c$ & 2 & 0.38 & ns & 0.01 & $\mathrm{~ns}$ & 0.18 & ns \\
\hline Factores vs Adicionales & 1 & 1.47 & ns & 17.73 & $* *$ & 50.05 & $* *$ \\
\hline Testigo o vs Ti & 1 & 0.20 & ns & 1.74 & ns & 1.83 & $\mathrm{~ns}$ \\
\hline Repeticiones & 3 & 1.22 & ns & 2.59 & $\mathrm{~ns}$ & 1.98 & ns \\
\hline Error & 39 & & & & & & \\
\hline Coeficiente de Variación & $\%)$ & 20.09 & & 23.46 & & 25.38 & \\
\hline
\end{tabular}

Cuadro 8. Análisis de varianza para el rendimiento en hoja de Stevia rebaudiana, durante tres cortes productivos.

El rendimiento de hoja seca de Stevia a medida que la planta se hace longeva, incrementa su producción en parte por regeneración vegetativa de acuerdo con los resultados del análisis estadístico del ensayo. La influencia de las fermentaciones de biol (factor a) en conjunto con las coberturas inertes (factor b) en evaluación con las densidades de siembra (factor $\mathrm{c}$ ) de esta variable se hace notoria en el segundo y tercer corte productivo de Stevia, con un alta significancia estadística en comparación con los testigos. Hay influencia sobre el comportamiento agroproductivo de la Stevia.

En el Cuadro 9 se observa el análisis de la prueba de Tukey al $5 \%$ de probabilidad, para rendimiento en hoja. Según los resultados obtenidos durante el segundo corte del cultivo, hay diferencia estadísticas entre los tratamientos. Los mejores rendimientos en producción de hoja seca, evaluados en 100 plantas se encuentran: T11 (Biol a los 75 días con Aserrín más densidad doble hilera) 644,10 gramos hoja seca/10o pl., T7 (Biol a los 60 días con Aserrín más densidad doble hilera) con 622,50 gramos hoja seca/100 pl. y T9 (Biol a los 75 días con C de arroz más densidad triple hilera) con promedio de 586 gramos hoja seca/100 pl.; siendo estadísticamente iguales. A diferencia de los testigos (T13 y T14), con los valores promedios más bajos de 389.90 y 281.50 gramos hoja seca/100 pl. en rendimiento de hoja seca.

De acuerdo a los resultados de Tukey $5 \%$, obtenidos durante la tercera cosecha del cultivo, reporta tres rangos de significancia 
estadística, en el primer rango se ubican el T11 (Biol a los 75 días con aserrín más densidad doble hilera) con una media de 1137.80 gramos hoja seca/100 pl. y T1 (Biol a los 45 días con C de arroz más densidad doble hilera) de 1101.80 gramos hoja seca/100 pl. Mientras que T13 (testigo doble hilera) se mantiene en el último rango con una producción de hoja seca de 260.80 gramos hoja seca/100 pl.

Santamaría, 2009 reporta que "El biofertilizante líquido enriquecido es rico en minerales, aminoácidos, vitaminas y hormonas. También mejora el balance nutricional en la planta, haciéndola mas resistente al ataque de plagas y enfermedades originadas por el desequilibrio ambiental; es por eso que en algunos casos se le atribuye el efecto de actuar como repelente, fungicida o insecticida suave".

\begin{tabular}{|c|c|c|c|c|c|c|c|c|c|}
\hline \multicolumn{4}{|c|}{ Tratamientos } & \multicolumn{6}{|c|}{ Rendimiento hoja seca/100 plantas (gramos) } \\
\hline \# & Biol & Cobertura & Densidad & Primera cose & & Segunda $\cos$ & cha & Tercera cose & \\
\hline 1 & 45 días & C. de arroz & doble & 169.60 & a & 412.00 & $a b$ & 1101.80 & a \\
\hline 2 & 45 días & C. de arroz & triple & 155.10 & $\mathrm{a}$ & 436.00 & $a b$ & 891.30 & $a b$ \\
\hline 3 & 45 días & Aserrín & doble & 165.90 & a & 537.70 & $a b$ & 931.80 & $a b$ \\
\hline 4 & 45 días & Aserrín & triple & 169.10 & a & 470.70 & $a b$ & 865.50 & $a b$ \\
\hline 5 & 60 días & C. de arroz & doble & 179.10 & a & 504.80 & $a b$ & 1002.00 & $a b$ \\
\hline 6 & 60 días & C. de arroz & triple & 190.90 & a & 499.80 & $a b$ & 1016.50 & $a b$ \\
\hline 7 & 60 días & Aserrín & doble & 177.80 & $\mathrm{a}$ & 622.50 & $\mathrm{a}$ & 976.30 & $a b$ \\
\hline 8 & 60 días & Aserrín & triple & 170.60 & $\mathrm{a}$ & 529.40 & $a b$ & 975.50 & $a b$ \\
\hline 9 & 75 días & C. de arroz & doble & 171.60 & $\mathrm{a}$ & 586.30 & a & 981.80 & $a b$ \\
\hline 10 & 75 días & C. de arroz & triple & 153.20 & $\mathrm{a}$ & 542.20 & $a b$ & 899.80 & $a b$ \\
\hline 11 & 75 días & Aserrín & doble & 198.90 & $\mathrm{a}$ & 644.10 & $\mathrm{a}$ & 1137.80 & a \\
\hline 12 & 75 días & Aserrín & triple & 162.50 & a & 489.00 & $a b$ & 1029.80 & $a b$ \\
\hline 13 & Testigo 1 & & doble & 150.90 & $\mathrm{a}$ & 389.90 & b & 260.80 & c \\
\hline 14 & Testigo 2 & & triple & 161.60 & $\mathrm{a}$ & 281.50 & $\mathrm{~b}$ & 478.30 & $\mathrm{bc}$ \\
\hline
\end{tabular}

Cuadro 9. Análisis de Tukey al $5 \%$ para la variable rendimiento hoja seca/100 plantas (gr) Stevia rebaudiana, durante tres cortes productivos.

Las fermentaciones de biol (factor a) presenta diferencias significativas en el rendimiento a la segunda cosecha de Stevia rebaudiana. El biol fermentado a los 75 días (a3) tiene el mayor efecto en el rendimiento productivo, con un promedio de 565.40 gramos hoja seca/100 pl. siendo superior al resto. Según Tukey al 5 $\%$ el biol fermentado a los 45 días (a1) tiene la menor influencia en la producción de hoja de Stevia con 464.10 gramos hoja seca/100 pl. (Figura 7).

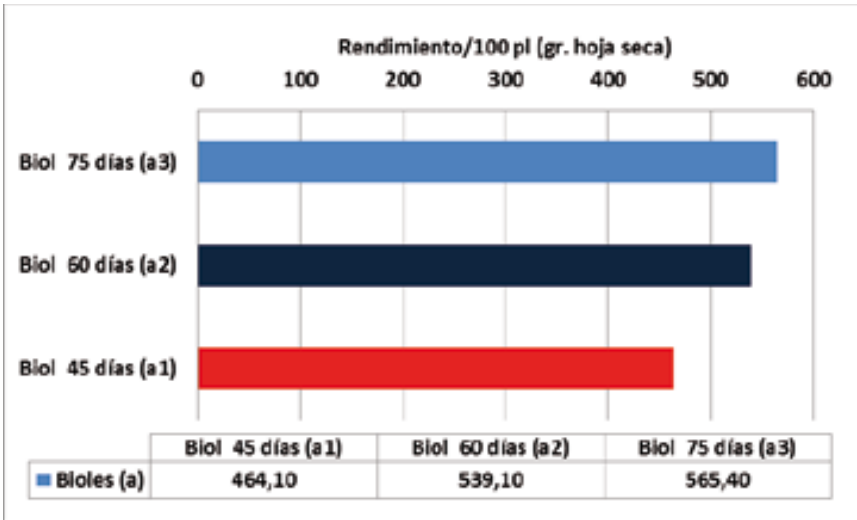

Figura 7. Influencia de las fermentaciones de Biol en el segundo corte productivo de Stevia rebaudiana.

En la Figura 8 se observan la respuesta de los tiempos de fermentación del biol, durante el desarrollo productivo de la Stevia rebaudiana. A partir de la segunda cosecha del cultivo, los tiempos de fermentación presentan incidencia perceptible en el rendimiento de hoja seca. La fermentación de biol a 75 días, presenta los mejores promedios productivos durante el ciclo del cultivo con 137.24 KgHsha-1 (primera cosecha), 452.31 KgHsha-1 (segunda cosecha) y 809.80 KgHsha-1 (tercera cosecha). El efecto de las fermentaciones se hace notable al observarse el rendimiento de los testigos dentro de la investigación con una producción mínima de 120.74 a 129.26 KgHsha-1 (primer corte), 311.92 a 225.16 KgHsha-1 (segundo corte) y 208.60 a $382.60 \mathrm{KgHsha}-1$ (tercer corte).

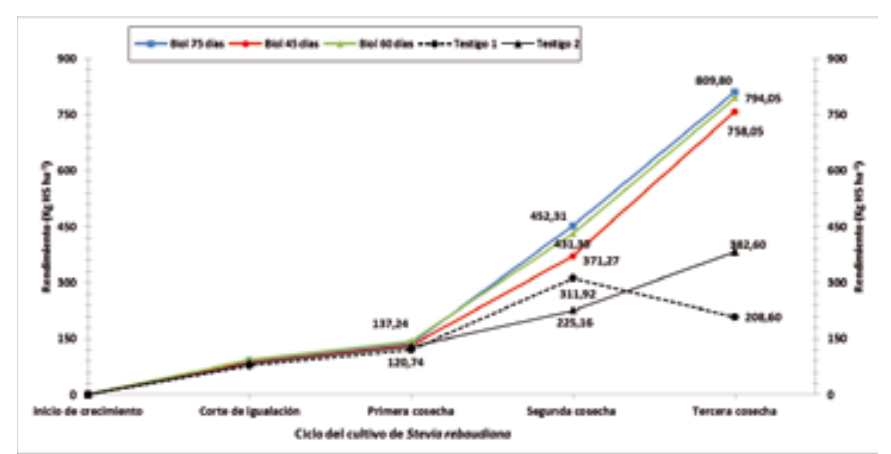

Figura 8. Influencia de los tiempos de fermentación de biol en el rendimiento de Stevia rebaudiana, durante el ciclo de crecimiento. 
En la Figura 9, muestra el rendimiento en hoja seca, bajo las densidades de siembra en el cultivo de Stevia. La arquitectura de planta permite la siembra en altas densidades de acuerdo con los resultados de esta investigación, no muestran diferencias aparentes en el rendimiento de hoja seca. La densidad tripe hilera, mantiene los promedios más altos con 166.91 KgHsha-1 (Primera cosecha), 494.53 KgHsha-1 (Segunda cosecha) y 946.38 KgHsha-1 (Tercera cosecha) respectivamente.

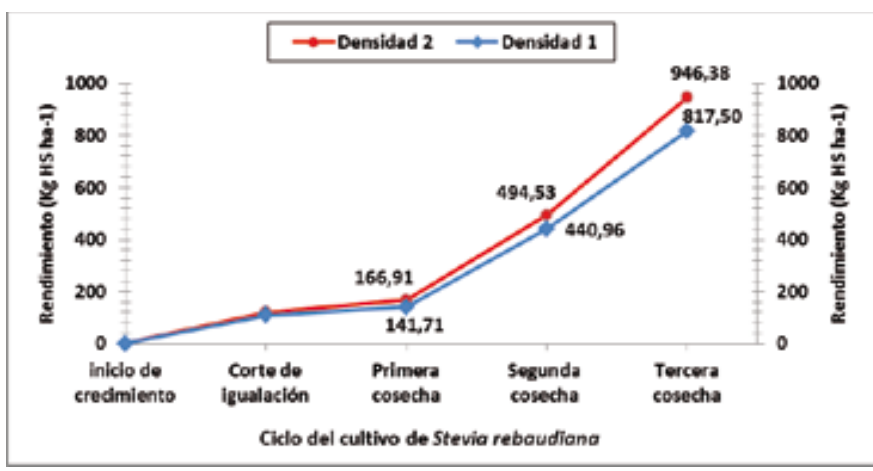

Figura 9. Rendimiento de Stevia rebaudiana, bajo dos densidades de siembra, en el ciclo de crecimiento.

Alcívar y Parraga, 2012 citan que "El uso de biol enriquecido, considerado así por la incorporación minerales y microorganismos aplicados tanto al follaje de las cultivos como al suelo, favorece el desarrollo vegetativo y el incremento de la producción de las mismos. El rendimiento de los cultivos tiende a ser mayor a medida que se incrementan las dosis de biol al follaje".

\section{Incidencia de Malezas (malezas $\mathrm{m}^{-2}$ )}

El Cuadro 10 presenta el análisis de varianza para la incidencia de malezas en el cultivo durante las tres cosechas de Stevia rebaudiana. Para el análisis estadístico de esta variable, se sometió a transformación de raíz cuadrada $(\mathrm{V} \mathrm{x})$ con el fin de detectar diferencias reales entre las unidades experimentales. Para los tratamientos evaluados, presentó alta significancia estadística, evaluados en los tres cortes productivos del cultivo. Mientras que para las coberturas inertes (factor b) presentan diferencias a partir de la segunda y tercera cosecha. Los coeficientes de variación fueron $16.51 \%, 17.57$ \% y $18.50 \%$, siendo aceptables en la investigación.

\begin{tabular}{|c|c|c|c|c|c|c|c|}
\hline \multirow{2}{*}{$\begin{array}{l}\text { F.V. } \\
\text { Total }\end{array}$} & \multirow{2}{*}{$\begin{array}{l}\mathrm{gl} \\
55\end{array}$} & \multicolumn{2}{|c|}{ Primera cosecha } & \multicolumn{2}{|c|}{ Segunda cosecha } & \multicolumn{2}{|c|}{ Tercera cosecha } \\
\hline & & & & & & & \\
\hline Tratamientos & 13 & 49.11 & $* *$ & 24.03 & $* *$ & 19.42 & $* *$ \\
\hline Bioles (a) & 2 & 1.35 & ns & 2.06 & ns & 1.84 & ns \\
\hline Coberturas (b) & 1 & 0.20 & ns & 11.29 & $* *$ & 8.27 & ** \\
\hline$a * b$ & 2 & 0.84 & ns & 0.23 & ns & 0.40 & $\mathrm{~ns}$ \\
\hline Densidades $(C$ & 1 & 0.25 & ns & 0.02 & ns & 0.05 & ns \\
\hline$a^{*} c$ & 2 & 0.92 & ns & 1.01 & ns & 1.43 & ns \\
\hline$b^{*} \mathrm{c}$ & 1 & 0.12 & ns & 1.08 & ns & 0.03 & $\mathrm{~ns}$ \\
\hline$a^{*} b^{*} c$ & 2 & 0.04 & ns & 1.26 & ns & 0.15 & ns \\
\hline Factores vs Adicionales & 1 & 630.64 & $* *$ & 290.78 & $* *$ & 234.22 & $* *$ \\
\hline Testigo o vs Ti & 1 & 0.99 & ns & 0.05 & ns & 2.28 & $\mathrm{~ns}$ \\
\hline Repeticiones & 3 & 13.24 & & 7.77 & & 14.57 & \\
\hline Error & 39 & & & & & & \\
\hline Coeficiente de Variación & $\%)$ : & 16.51 & & 17.57 & & 18.50 & \\
\hline
\end{tabular}

Cuadro 10. Análisis de varianza para la incidencia de malezas en Stevia rebaudiana, durante tres cortes productivos.

El uso de las coberturas inertes es eficaz en el control de malezas del cultivo, en conjunto con las fermentaciones de biol y las densidades de siembra, muestran diferencias estadísticas en comparación con los testigos que se probaron en esta investigación. Las coberturas evitan la competencia directa con las malezas, favoreciendo al cultivo en su desarrollo fisiológico con el fin de mejorar su rendimiento y lo más primordial evitando la pérdida de partículas de suelo.

En el Cuadro 11, se observa el análisis de la prueba de Tukey al $5 \%$ de probabilidad, para la incidencia de malezas en la Stevia. Según los resultados obtenidos durante los tres cortes productivos se detectó dos rangos significativos. Los testigos (T13 y T14) presentaron la mayor incidencia de malezas en el ciclo de cultivo de la Stevia rebaudiana con promedios que oscilan entre 215.13 a 236.88 malezas $/ \mathrm{m}^{2}$ (primera cosecha), 105.75 a 109.25 malezas $/ \mathrm{m}^{2}$ (segunda cosecha) y 244.75 a 203.13 malezas/ $\mathrm{m}^{2}$ (tercera cosecha). En el último rango estadístico se ubicaron el resto de tratamientos, que reportan la menor proliferación de malezas por $\mathrm{m}^{2}$, con promedios de 18.88 a 36.63 malezas $/ \mathrm{m}^{2}$ (primera cosecha), 12.25 a 30.00 malezas $/ \mathrm{m}^{2}$ (segunda cosecha) y la última cosecha, la incidencia estuvo entre 28.63 y 65.63 malezas $/ \mathrm{m}^{2}$. 
Las coberturas inertes (Factor b) presenta influencia en el control de malezas durante el desarrollo productivo de la Stevia rebaudiana. El efecto de las diferentes coberturas es altamente significativo en el segundo y tercer corte productivo de la planta. Según Tukey al 5 \%, el uso de aserrín de madera (b2) presento mayor incidencia de malezas con valores promedio que van de 24.71 a 52.38 malezas/m-2. A diferencia del cobertura de Cascarilla de Arroz (b1) que ejerció mayor control en las malezas con una proliferación de 16,08 a 36,48 malezas/m-2.

Ortiz, 2004, argumenta que “Las cubiertas de materia orgánica inerte, constituyen un buen método para el control de malezas y para mantener las plantas saludables. Una capa gruesa de mulching mantiene el suelo aislado, permite conservar la humedad (al reducir la evaporación), impide la erosión, evita que el follaje toque el suelo (lo que disminuye el riesgo de enfermedades de las plantas que se transmiten a través de éste) y mantiene las plagas alejadas. Cuando se descompone, el mulching incorpora humus y nutrientes al suelo. Hay que tener cuidado de que la capa de mulching no sea demasiado gruesa, ya que la misma podría ahogar el suelo, impidiendo la circulación de aire, que es de vital importancia. Para ello, es necesario que haya varias pulgadas de distancia entre el mulching y la base de la planta, lo cual, a su vez, evita la pudrición y dificulta el acceso de las plagas".

\begin{tabular}{llll|rlrlrl}
\hline \multicolumn{9}{c|}{ Tratamientos } & \multicolumn{5}{c}{ Incidencia de malezas (\# malezas m ${ }^{-2}$ ) } \\
\hline \# & Biol & Cobertura & Densidad & Primera cosecha & Segunda cosecha & Tercera cosecha \\
\hline 1 & 45 días & C. de arroz & doble & 31.00 & $\mathrm{~b}$ & 18.13 & $\mathrm{~b}$ & 44.00 & $\mathrm{~b}$ \\
2 & 45 días & C. de arroz & triple & 36.63 & $\mathrm{~b}$ & 17.88 & $\mathrm{~b}$ & 48.13 & $\mathrm{~b}$ \\
3 & 45 días & Aserrín & doble & 22.50 & $\mathrm{~b}$ & 30.00 & $\mathrm{~b}$ & 52.00 & $\mathrm{~b}$ \\
4 & 45 días & Aserrín & triple & 27.25 & $\mathrm{~b}$ & 28.75 & $\mathrm{~b}$ & 65.63 & $\mathrm{~b}$ \\
5 & 60 días & C. de arroz & doble & 24.63 & $\mathrm{~b}$ & 15.25 & $\mathrm{~b}$ & 28.63 & $\mathrm{~b}$ \\
6 & 60 días & C. de arroz & triple & 30.75 & $\mathrm{~b}$ & 16.88 & $\mathrm{~b}$ & 30.38 & $\mathrm{~b}$ \\
7 & 60 días & Aserrín & doble & 24.25 & $\mathrm{~b}$ & 21.38 & $\mathrm{~b}$ & 51.13 & $\mathrm{~b}$ \\
8 & 60 días & Aserrín & triple & 26.00 & $\mathrm{~b}$ & 25.63 & $\mathrm{~b}$ & 49.63 & $\mathrm{~b}$ \\
9 & 75 días & C. de arroz & doble & 21.00 & $\mathrm{~b}$ & 12.25 & $\mathrm{~b}$ & 38.25 & $\mathrm{~b}$ \\
10 & 75 días & C. de arroz & triple & 18.88 & $\mathrm{~b}$ & 16.13 & $\mathrm{~b}$ & 29.50 & $\mathrm{~b}$ \\
11 & 75 días & Aserrín & doble & 26.25 & $\mathrm{~b}$ & 26.63 & $\mathrm{~b}$ & 54.13 & $\mathrm{~b}$ \\
12 & 75 días & Aserrín & triple & 22.00 & $\mathrm{~b}$ & 15.88 & $\mathrm{~b}$ & 41.75 & $\mathrm{~b}$ \\
13 & Testigo 1 & doble & 215.13 & $\mathrm{a}$ & 105.75 & $\mathrm{a}$ & 244.75 & $\mathrm{a}$ \\
14 & Testigo 2 & triple & 236.88 & $\mathrm{a}$ & 109.25 & $\mathrm{a}$ & 203.13 & $\mathrm{a}$ \\
\hline
\end{tabular}

Cuadro 11. Análisis de Tukey al $5 \%$ para la variable incidencia de malezas para Stevia rebaudiana, durante tres cortes productivos.
De acuerdo con la figura 10, las coberturas inertes (factor b) presentaron la menor proliferación de malezas durante el ciclo de cultivo de la Stevia rebaudiana, la coberturas inertes (factor b) ejercieron control eficiente en la incidencia de malezas en el cultivo. En la primera cosecha el control de malezas entre las coberturas de Cascarilla de Arroz (b1) y Aserrín (b2) no hay diferencias estadísticas con valores de 27.15 y 24.71 malezas/m-2.En la segunda y tercera cosecha el efecto de la Cascarilla de Arroz se hace evidente en la incidencia de malezas en un rangos de 16,08 a 36,48 malezas/m-2. Los tratamientos Testigo reportan la dinámica de las malezas a lo largo del ciclo productivo de la Stevia rebaudiana, con la proliferaciones más altas en el primero (236.87 y 215.12 malezas/m-2) y tercer corte productivo de la planta con promedios de 244.75 y 203.13 malezas/m-2; en el segundo corte productivo se presento los valores de proliferación más baja con 109,25 malezas/m-2.

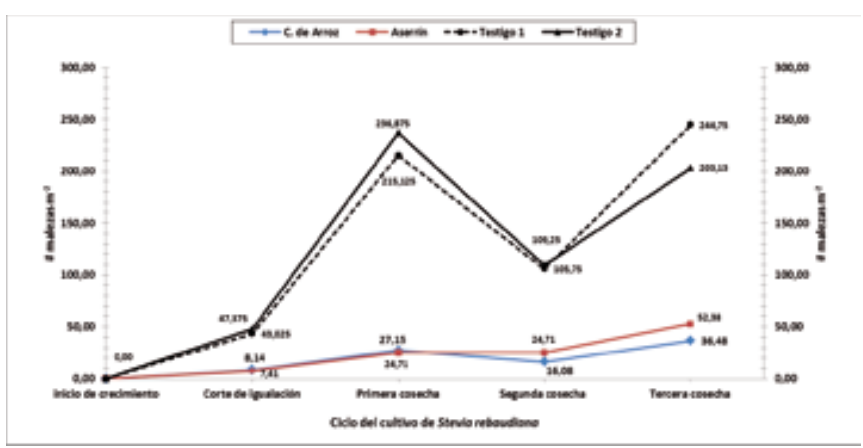

Figura 10. Influencia de las coberturas inertes en la dinámica poblacional de las malezas en el ciclo de cultivo de Stevia rebaudiana.

\section{Monitoreo de Plagas y Enfermedades}

\section{Plagas}

La hoja de Stevia rebaudiana es el órgano de importancia económica, este es un indicador clave de la adaptación de la planta a la zona. Uno de los factores que incide negativamente en el rendimiento y calidad del producto, es la presencia de insectos que causan daños considerables. Fue necesario identificar el tipo de ataque que se presento durante todo el ciclo del cultivo. De acuerdo 
con el monitoreo se identificó a tres insectos plaga que incidieron en el desarrollo de las plantas evaluados en el ensayo (Cuadro 12).

\begin{tabular}{llll}
\hline Nombre común & Nombre científico & Orden & Órgano atacado \\
\hline Grillo topo & Gryllotalpa $\boldsymbol{s} \boldsymbol{p}$ & Orthoptera & Raíz-tallo \\
Cochinilla & Pseudococcus $\boldsymbol{s p}$ & Homóptera & Raíz \\
Trips & Trips tabaco & Thysanoptera & Hojas-brotes \\
\hline
\end{tabular}

Cuadro 12. Plagas que atacan al cultivo de Stevia rebaudiana en Santo Domingo.

En el Cuadro 13, se reportan la incidencia de plagas, presentes en las tres cosechas productivas de Stevia rebaudiana. La proliferación de esta plaga fue masiva en los tratamientos testigo (T13, T14), al presentar los factores que inciden negativamente en el rendimiento y calidad de la planta, en especial la especie del orden Homóptera causan un daño indirecto, pues se alimentan de la savia o del jugo celular, disminuyendo el crecimiento de la planta por reducción de fotoasimilados disponibles para los procesos metabólicos. Mientras que el resto de tratamientos la presencia de plagas fue de bajo impacto sin necesidad de llevar un control estricto en el ciclo del cultivo, con un promedio del 20\% de afección del cultivo.

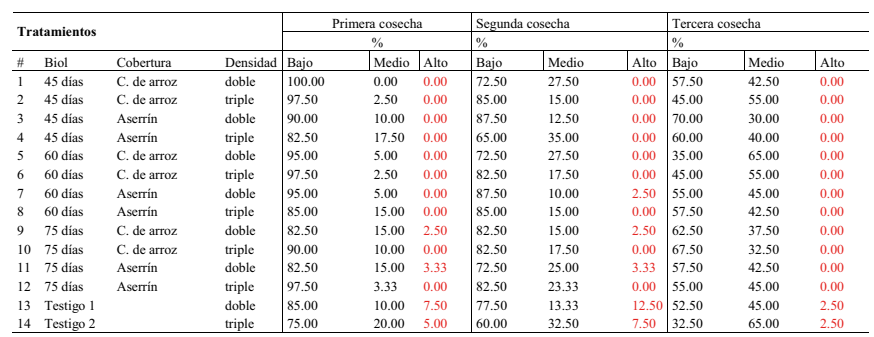

Cuadro 13. Incidencia de la proliferación de plagas, durante el ciclo de productivo de Stevia rebaudiana

\section{Enfermedades}

En el Cuadro 14 se observa las enfermedades fúngicas, que afectaron el desarrollo normal del cultivo de Stevia rebaudiana. La determinación de las enfermedades fúngicas se hizo mediante monitoreos en el cultivo, durante los tres cortes productivos. Los síntomas presente en la plantación fue descrita visualmente y se recolectaron muestras para ser aisladas en el Laboratorio de Sanidad Vegetal del INIAP para su identificación respectiva. Según los resultados obtenidos podemos indicar, que con un $70 \%$ de frecuencia se mostró el género Rhizoctonia sp es causante de la pudrición blanca del cuello de la planta, en tanto que con una frecuencia de un $20 \%$ se encontró al género Fusarium sp, hongo patógeno del suelo, causante del ataque a nivel radicular ocasionando la muerte descendente de las plantas de Stevia.

\begin{tabular}{|c|c|c|c|c|}
\hline Metodología & Tipo & Parte analizada & Organismo a identificar & Frecuencia $(\%) * *$ \\
\hline \multirow[t]{3}{*}{ PDA - CMA y LCH * } & Hongos & Cuello de la raíz & Rhizoctonia sp & 70 \\
\hline & & & Fusarium sp & 20 \\
\hline & & & Trichoderma sp & 10 \\
\hline \multirow[t]{3}{*}{ PDA - CMA y LCH * } & Hongos & Sistema radical & Cylindrocarpon sp & 50 \\
\hline & & & Trichoderma sp & 30 \\
\hline & & & Fusarium $s p$ & 20 \\
\hline
\end{tabular}

A = Corn Meal Agar $\quad$ LCH = Lactosa Caseína hidrolizada

** \% de aislamientos en medios de cultivos de cada organismo recuperado

Cuadro 14. Identificación de agentes fúngicos causantes de enfermedades en el cultivo de Stevia rebaudiana

Fusarium sp y Rhizoctonia sp; se encuentran reportados como patógenos en el cultivo de Stevia. Cylindrocarpon sp, es un hongo que se viene aislando con frecuencia en problemas radicales de otros cultivos. Trichoderma sp, es un hongo antagonista del suelo, y se ha encontrado debido a las aplicaciones preventivas que se realizó con dicho hongo en los estadios iniciales de la Stevia rebaudiana. La incidencia de enfermedades fue masiva en los tratamientos T13 y T14 (Testigos), en el tercer corte productivo, con un promedio alto de daño entre el 40,00 y $43.33 \%$, reduciendo de esta manera la población inicial de plantas por unidad de área a diferencia del resto de tratamientos que tuvo baja proliferación de enfermedades causados por estos patógenos y el control sanitario se hizo evidente como un medio preventivo que favoreció el desarrollo optimo del cultivo (Cuadro 15). 


\begin{tabular}{|c|c|c|c|c|c|c|c|c|c|c|c|c|}
\hline \multirow{2}{*}{\multicolumn{4}{|c|}{ Tratamientos }} & \multirow{2}{*}{\multicolumn{3}{|c|}{$\begin{array}{c}\text { Primera cosecha } \\
\%\end{array}$}} & \multirow{2}{*}{\multicolumn{3}{|c|}{ Segunda cosecha }} & \multicolumn{3}{|c|}{ Tercera cosecha } \\
\hline & & & & & & & & & & & $\%$ & \\
\hline$\#$ & Biol & Cobertura & Densidad & Bajo & Medio & Alto & Bajo & Medio & Alto & Bajo & Medio & Alto \\
\hline 1 & 45 dias & C. de arroz & doble & 77.50 & 23.33 & 6.67 & 65.00 & 25.00 & 10.00 & \begin{tabular}{|l|l|}
60.00 \\
\end{tabular} & 43.33 & 0.00 \\
\hline 2 & 45 dias & C. de arroz & & 85.00 & 16.67 & 3.33 & 75.00 & 2000 & 5.00 & 53.33 & 40.00 & 6.67 \\
\hline 3 & 45 dias & Aserrín & doble & 82.50 & 12.50 & 10.00 & 75.00 & 15.00 & 20.00 & 46.67 & 46.67 & 6.67 \\
\hline 4 & 45 días & Aserrín & triple & 62.50 & 35.00 & 5.00 & 50.00 & 45.00 & 10.00 & 66.67 & 26.67 & 6.67 \\
\hline 5 & 60 & C. de & & 75.00 & 25.00 & 0.00 & 65.00 & & 0.00 & 63.33 & & 0.00 \\
\hline 6 & 60 dias & C. de arroz & trip & 67.50 & 33.33 & 10.00 & 45.00 & 40.00 & 15.00 & 50.00 & 46.67 & 3.33 \\
\hline 7 & 60 dias & Aserrin & & 92.50 & 10.00 & 0.00 & 90.00 & 10.00 & 0.00 & 56.67 & 43.33 & 0.00 \\
\hline 8 & 60 día & Aserrin & tripl & 65.00 & 32. & 3.33 & 65.00 & 30.00 & 5.0 & 53.33 & 77 & 0.00 \\
\hline 9 & 75 días & C. de arr & we & 62.50 & 32. & 6.67 & 50.00 & 40.00 & 10.00 & 43.33 & 56.67 & 0.00 \\
\hline 10 & 75 dias & C. de arr & 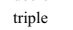 & 65.00 & 40.00 & 6.67 & 35.00 & 55.00 & 10.00 & 40.00 & 56.67 & 3.33 \\
\hline 11 & 75 días & Aserri & dobl & 70.00 & 27.5 & 3.3 & 65.00 & 30.0 & 5.0 & 53.33 & 46.67 & 0.00 \\
\hline 12 & 75 dias & Aserrin & triple & 85.00 & 30.00 & 0.00 & 75.00 & 50.00 & 0.00 & 56.67 & 43.33 & 0.00 \\
\hline 13 & Testigo 1 & & doble & 77.50 & 23.33 & 10.00 & 60.00 & 30.00 & 20.00 & 16.67 & 43.33 & 40.00 \\
\hline 14 & Testigo 2 & & ple & 85.00 & 15.00 & 10.00 & 75.00 & 20.00 & 15.00 & 26.67 & 30.00 & 43.33 \\
\hline
\end{tabular}

Cuadro 15. Incidencia de la proliferación de enfermedades, durante el ciclo de productivo de Stevia rebaudiana

Concentración de Nutrientes en la hoja de Stevia rebaudiana

\begin{tabular}{|c|c|c|c|c|c|c|c|c|c|c|c|c|}
\hline \multirow{2}{*}{\multicolumn{4}{|c|}{ Tratamientos }} & \multirow{2}{*}{\multicolumn{9}{|c|}{\begin{tabular}{c|c|c} 
Primera cosecha & Segunda cosecha & Tercera cos \\
\% DE MATERIA SECA
\end{tabular}}} \\
\hline & & & & & & & & & & & & \\
\hline \# & Biol & Cobertura & Densidad & $\mathrm{N}$ & $\mathrm{P}$ & $\mathrm{K}$ & $\mathrm{N}$ & $P$ & $\mathrm{~K}$ & $\mathrm{~N}$ & $P$ & $\mathrm{~K}$ \\
\hline 1 & 45 días & C. de arroz & doble & 0.92 & 0.08 & 3.95 & 1.31 & 0.11 & 3.24 & 1.70 & 0.14 & 2.53 \\
\hline 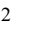 & 45 días & C. de arroz & triple & 0.76 & 0.08 & 4.10 & 1.19 & 0.15 & 2.93 & 1.62 & 0.21 & 1.75 \\
\hline 3 & 45 días & Aserrín & doble & 0.92 & 0.20 & 4.73 & 1.25 & 0.20 & 4.97 & 1.57 & 0.19 & 5.20 \\
\hline 4 & 45 días & Aserrín & triple & 1.29 & 0.10 & 5.03 & 1.37 & 0.15 & 4.87 & 1.45 & 0.20 & 4.70 \\
\hline 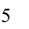 & 60 días & C. de arroz & doble & 1.23 & 0.13 & 4.15 & 1.41 & 0.11 & 3.93 & 1.58 & 0.08 & 3.70 \\
\hline 6 & 60 días & C. de arroz & triple & 0.87 & 0.08 & 4.63 & 1.14 & 0.10 & 4.42 & 1.40 & 0.12 & 4.20 \\
\hline 7 & 60 días & Aserrín & doble & 1.04 & 0.06 & 4.50 & 1.31 & 0.13 & 4.15 & 1.57 & 0.19 & 3.80 \\
\hline 8 & 60 días & Aserrín & triple & 1.29 & 0.06 & 6.30 & 1.32 & 0.12 & 5.84 & 1.34 & 0.17 & 5.38 \\
\hline . & 75 días & C. de arroz & doble & 2.07 & 0.11 & 6.30 & 1.81 & 0.10 & 4.90 & 1.54 & 0.08 & 3.50 \\
\hline 10 & 75 días & C. de arroz & triple & 2.13 & 0.10 & 7.25 & 1.73 & 0.15 & 5.22 & 1.32 & 0.19 & 3.18 \\
\hline 11 & 75 días & Aserrín & doble & 1.82 & 0.10 & 6.18 & 1.65 & 0.13 & 3.99 & 1.48 & 0.16 & 1.80 \\
\hline 12 & 75 días & Aserrín & triple & 1.90 & 0.79 & 3.75 & 1.55 & 0.44 & 3.57 & 1.20 & 0.09 & 3.38 \\
\hline 13 & Testigo 1 & & doble & 1.96 & 0.06 & 4.40 & 1.82 & 0.09 & 3.17 & 1.68 & 0.11 & 1.93 \\
\hline 14 & Testigo 2 & & triple & 1.88 & 0.08 & 5.38 & 1.64 & 0.16 & 3.89 & 1.40 & 0.23 & 2.40 \\
\hline
\end{tabular}

Cuadro 16. Concentración de N, P, K; en la hoja de Stevia rebaudiana, en tres cortes productivos

En el Cuadro 16 se observa la concentración de los principales macro nutrientes (NPK) presentes en la hoja seca de Stevia durante su ciclo productivo. La mayor tasa de absorción de Py K se reportó en la primera cosecha de Stevia en promedios que van en fósforo con 0.06 a $0.20 \%$ de materia seca y en potasio con rangos de 3.75 a $7.25 \%$ de materia seca. El nitrógeno $(\mathrm{N})$ se acumuló en niveles más altos en los tratamientos que se aplicaron, la fermentación de Biol a los 75 días de los T9, T10, T11 y T12 con una concentración que va de 1.82 a 2.13\% de materia seca.

En la segunda y tercera cosecha la concentración de NPK decrece en porcentaje de materia seca de la hoja. Los elementos empiezan a distribuirse uniformemente en los diferentes órganos a conforme la planta se torna longeva. Los niveles de nitrógeno y fósforo se mantienen estables en los diferentes tratamientos que se evaluaron en este ensayo en el segundo y tercer corte. A diferencia de los contenidos de Potasio (K), en la hoja que presenta diferencias altas entre los tratamientos y en comparación con los testigos ( $\mathrm{T} 13$, T14) debido a que el $\mathrm{K}$ es un activador de muchas enzimas esenciales para la fotosíntesis y la respiración, y también activa enzimas que son necesarias para formar almidón y proteínas. Es uno de los contribuyentes más importantes al potencial osmótico de las células y por consiguiente, a su presión de turgencia, por tanto el mecanismo estomático depende del flujo de iones K+.

\section{Conclusiones}

De acuerdo con los resultados se concluye que:

Los tratamientos T8 (Biol fermentado a los 60 días con cobertura de aserrín más densidad triple hilera) y T4 (Biol fermentado a los 45 días con cobertura de aserrín más densidad triple hilera) presentaron los promedios en altura de planta más aceptables en las tres cosechas de Stevia. Durante el primer corte el crecimiento fue de 40.05 cm y $39.38 \mathrm{~cm}$ respectivamente, en el segundo corte fue de 38.00 y 37.38 $\mathrm{cm}$ y en el último corte la altura estuvo entre 35.98 y $35.03 \mathrm{~cm}$.

Se presentó interacción entre las diferentes fermentaciones de biol (factor a) y las densidades de siembra (factor c) que determinaron la mejor respuesta sobre la densidad 2 (triple hilera), al utilizar las fermentación de biol a los 60 días, obtuvo un promedio de $39.08 \mathrm{~cm}$ de altura de planta y el mejor promedio de altura se consiguió con la densidad de siembra 2 (Triple hilera), que en el segundo corte de hoja de Stevia rebaudiana, obtuvo mayor relevancia con $38.10 \mathrm{~cm}$.

El tratamiento T5 (Biol fermentado a los 60 días con cobertura de $\mathrm{C}$ de arroz mas densidad doble hilera) presentó los 
mejores promedios en diámetro de tallo durante el desarrollo de esta investigación, obteniendo $0.82 \mathrm{~cm}$ (primera cosecha), $0.64 \mathrm{~cm}$ (segunda cosecha) y $0.92 \mathrm{~cm}$ (tercera cosecha).

Los factores fermentaciones de biol (factor a), coberturas inertes (factor b) y densidades de siembra (factor c), presentaron interacciones entre sí, en la variable diámetro de tallo en el segundo corte productivo de Stevia. La fermentación de biol a los 45 días influye significativamente en la amplitud del tallo en las diferentes densidades de siembra con un valor promedio de $0.70 \mathrm{~cm}$ en la densidad 1 (doble hilera). En tanto que el uso de la cobertura de cascarilla de arroz presenta los mejores valores en diámetro de tallo con la densidad 1 (doble hilera) con un promedio de $0.68 \mathrm{~cm}$ y $0.66 \mathrm{~cm}$.

El tratamiento T11 (Biol fermentado a los 75 días con cobertura de aserrín más densidad doble hilera) reporta el mayor índice en recuperación vegetativa durante el desarrollo de la investigación, con valores promedio de 7.58 brotes/ planta (primera cosecha), 27.58 brotes/planta (segunda cosecha) y 129.48 brotes/ planta (tercera cosecha).

Las fermentaciones de biol (factor a) se encuentran interactuando con las cobertura inertes (fractor b) y a la par con las densidades de siembra (factor $\mathrm{c}$ ), en el incremento de la recuperación vegetativa de la Stevia rebaudiana. Al aplicar fermentaciones de 60 días (a2) y 75 días (a3) la recuperación vegetativa de la Stevia es positiva con valores de 122.43 brote/planta a 129.25 brotes/ planta, con el uso de cobertura de cascarilla de arroz. En la aplicación de biol fermentado a los 75 días (az) la densidad 1 (doble hilera) reporta mayor incidencia con una formación de 129.56 brotes/planta.

En la producción de hoja seca de Stevia rebaudiana, el tratamiento T11 (Biol fermentado a los 75 días con cobertura de aserrín más densidad doble hilera) fue el mejor durante el ciclo productivo, sus valores promedio fueron: 198.90 gramos hoja seca/100 plantas (primera cosecha), 644.10 gramos hoja seca/100 plantas (segunda cosecha) y 1137.80 gramos hoja seca/100 plantas (tercera cosecha).

No hay presencia de interacciones entre los factores de estudio (a, b y c) que influyan en el rendimiento de hoja seca de Stevia. Las fermentaciones de biol inciden en el incremento productivo de materia seca, la fermentación de biol a los 75 días reportó los mejores valores con una producción de 137.24 KgHsha-1 (primera cosecha), 452.31 KgHsha-1 (segunda cosecha) y 809.80 KgHsha-1 (tercera cosecha).

Las coberturas inertes (factor b) influyen en el control de la dinámica poblacional de las malezas presentes en el cultivo de Stevia rebaudiana. La cascarilla de arroz (b1) presentó las mejores condiciones de control de malezas y conservación de la humedad, lo que permite mantener un ambiente favorable para el desarrollo de raíces, manteniendo la temperatura uniforme durante el ciclo del cultivo con valores promedios de proliferación de 27.15 malezas $/ \mathrm{m}^{2}$ en la primera cosecha, 16.08 malezas $/ \mathrm{m}^{2}$ en la segunda cosecha y en la última cosecha de Stevia, la incidencia fue 36.48 malezas $/ \mathrm{m}^{2}$.

La presencia de plagas y enfermedades en el cultivo de Stevia rebaudiana, fue intermitente durante el desarrollo del ensayo, teniendo ataques esporádicos que se mantuvieron por debajo del umbral económico. 


\section{Bibliografía}

Alcívar, Ey Parraga, F, 2012, Tesis de grado: Efecto del Biol enriquecido con bacterias acidolacticas en la productividad del cultivo de maní (Arachis hipogaea L), Escuela Superior Politécnica Agropecuaria de Manabí, Calceta - Ecuador, pág.3, 4, 10.

Amaya, X, 2010, Tesis de grado: efecto de tres densidades de siembra y tres dosis de bioinsecticidas en el cultivo de estevia (Eupatorium rebaudianum Bertoni) en la parroquia Tumbabiro - Cantón Urcuqui, Universidad Técnica del Norte Facultad de Ingeniería en Ciencias Agropecuarias y Ambientales, Ibarra - Ecuador, pág. 39.

Basantes, E, 2009, Tesis de grado: Elaboración y Aplicación de dos tipos de Biol en cultivo de Brócoli (Brassica oleracea Var. Legacy), Escuela Superior Politécnica de Chimborazo Facultad de Recursos Naturales Escuela de Ingeniería Agronómica, Riobamba-Ecuador, pág. 11, 12.

Birchler T, 1998. La planta ideal: Revisión del concepto, parámetros definitorios e implementación práctica. Nursery Technology Cooperative. Oregon State University Forest Sciences Lab 020. Corvallis. Oregon 97331 - 7501. EE.UU.

Cardoso E., Siu T. \& Neves M. 1992. Microbiología do Solo. Sociedade Brasileira de Ciencia do Solo. Campinas, Brasil

De la Cruz, R. 1997. Importancia del estudio biológico de las malezas. In: Seminario taller ciencia de las malezas. Guatemala, Guatemala. Centro Agronómico Tropical de Investigación y Enseñanza (CATIE). Proyecto regional del Manejo Integrado de Plagas (MIP). pp 69-86.

Guevara, A, 2011, Tesis de grado: Evaluación de Tres Abonos Líquidos Foliares Enriquecido con Micro elementos en la Producción Forrajera de una mezcla de Medicago sativa y Arrhenatherum, Escuela Superior Politécnica de Chimborazo Facultad de Ciencias Pecuarias Escuela de Ingeniería Zootécnica, Riobamba - Ecuador, pág. 7, 8.
Lindsey K. \& Jones M. 1992. Biotecnología Vegetal Agrícola. Editorial Acribia S.A. España.

Ministerio de Agricultura y Ganadería. 1996. Producción de Ka'a He'e. Tercera edición. República de Paraguay, Asunción. 35 p.

Ortiz, B, 2004, Tesis de grado: Efectos de cubiertas inertes sobre el control de malezas y crecimiento vegetativo del cerezo dulce (Prunus avium L.), bajo la modalidad de producción orgánica en el Secano Interior de la Comuna de Lumaco, IX Región de La Araucanía. Universidad Católica de Temuco, Facultad de Ciencias Agropecuarias y Forestales: Escuela de Agronomía, Temuco - Chile, pág. 37, 38.

Pitelli, R.A. 1995. Malezas en el sistema de siembra directa de cultivos anuales. Chile. 264 p.

Rivero, C. 1999. Revista alcance. Facultad de Agronomía, UCV. Vol. 57. 74, 75p.

Santamaria, D, 2009, Tesis de grado: Evaluación microbiana, hormonal y nutricional de ocho formulaciones en la preparación de biol y su aplicación en tres dosis en el cultivo de palmito (Bactris gasipaes HBK), Escuela Politécnica Del Ejército Facultad De Ciencias Agropecuarias I.A.S.A., Santo Domingo de los Tsáchilas - Ecuador, pág. 50, 55 y 56.

Savio, I y Zien, S. 2001. Weed Workshp. (En línea). Estados Unidos. (Fecha de consulta: 19 de noviembre, 2003). Disponible en: http://www.envtox.ucdavis.edu/cehs/TOXINS/SPANISH2 / herb icidesno.htm

Siquiera O. \& Franco A. 1988. Biotecnología del suelo. Fundamentos y perspectivas para las Ciencias Agrarias en los Trópicos Brasileños. Escuela Superior de Agricultura de Lavras, Brasil. 


\section{Anexos}
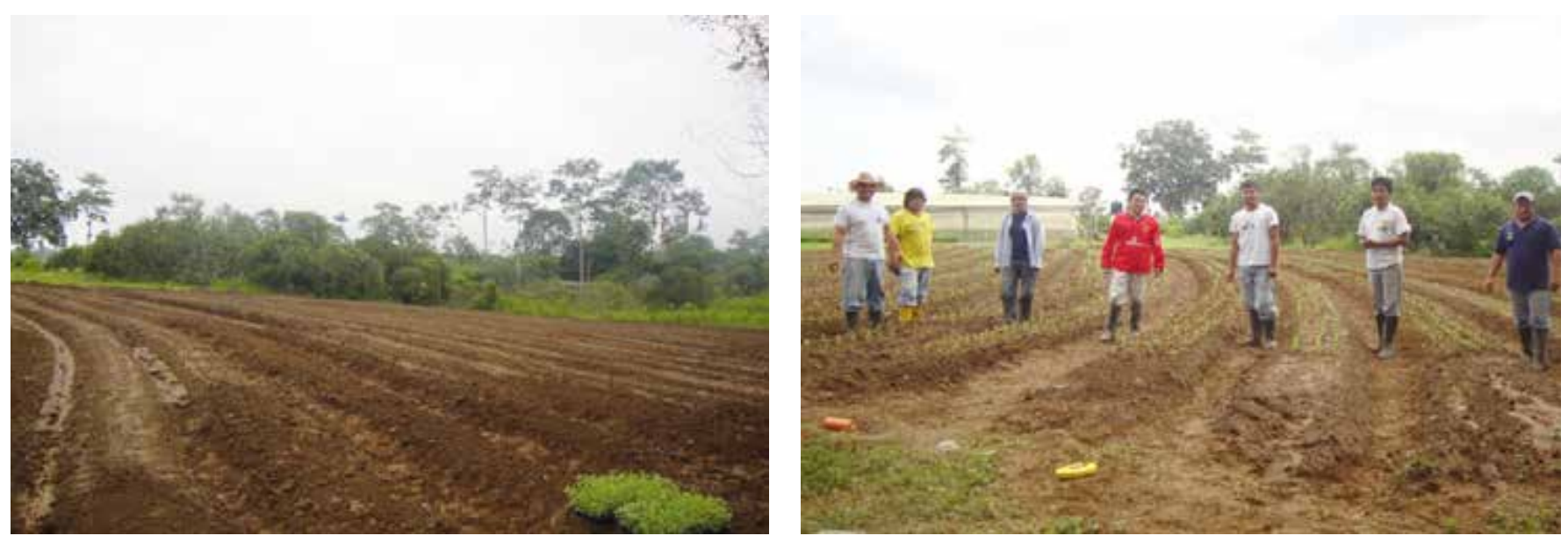

Foto $\mathrm{N}^{\circ}$ 1. Disposición de los tratamientos

Foto $\mathrm{N}^{\circ}$ 2. Siembra de plantines de Stevia
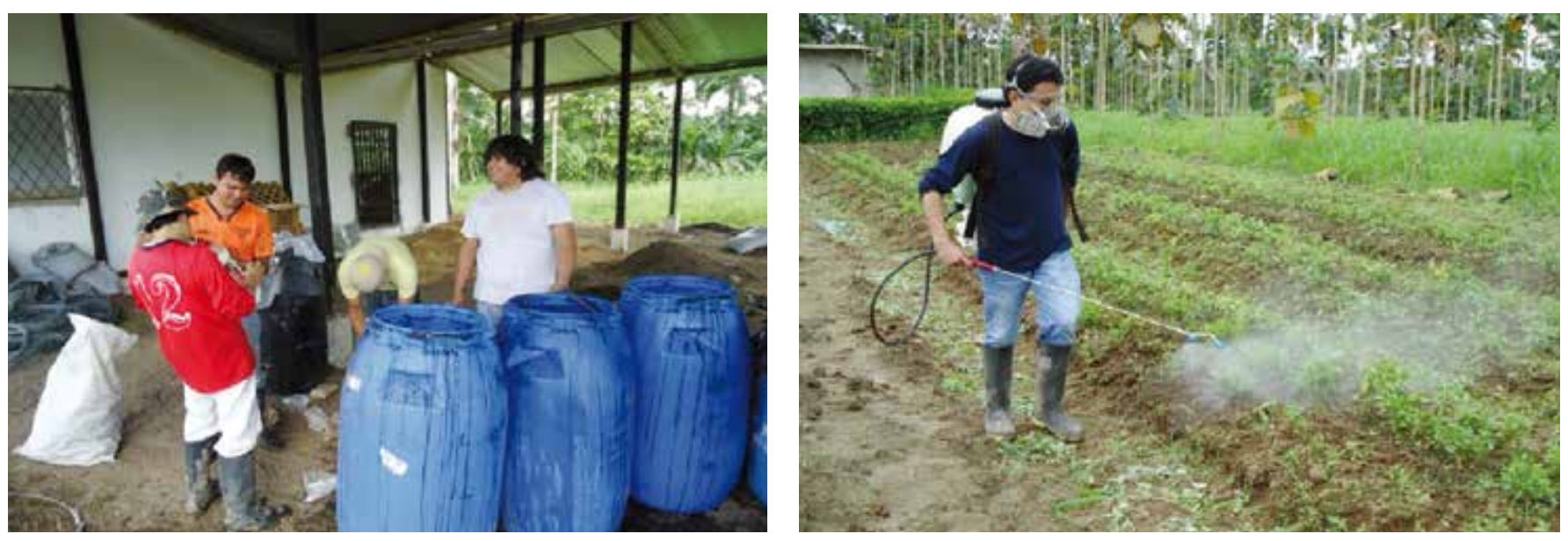

Foto $\mathrm{N}^{\circ}$ 3. Elaboración de bioles

Foto $\mathbf{N}^{\circ}$. Aplicación de bioles 


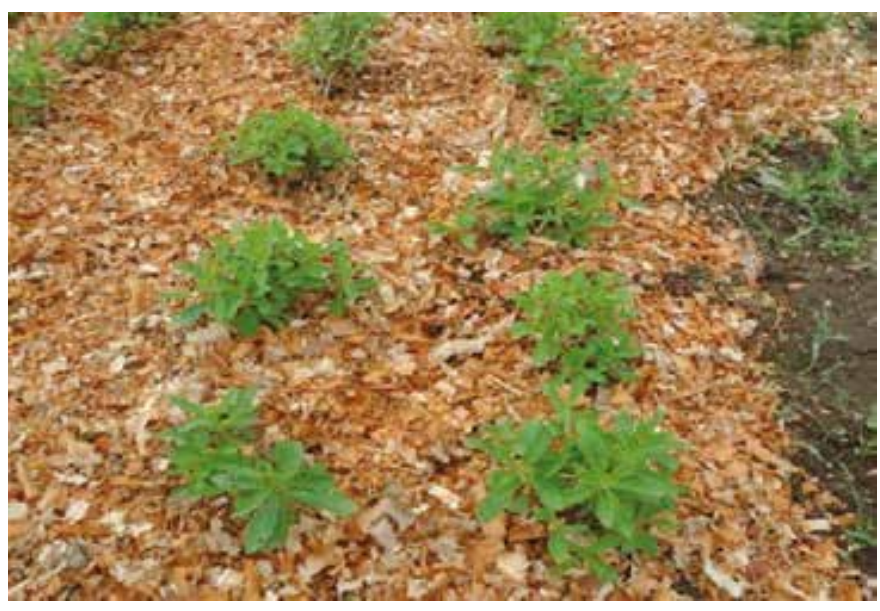

Foto $\mathrm{N}^{\circ} 5$. Aplicación de sustratos inertes

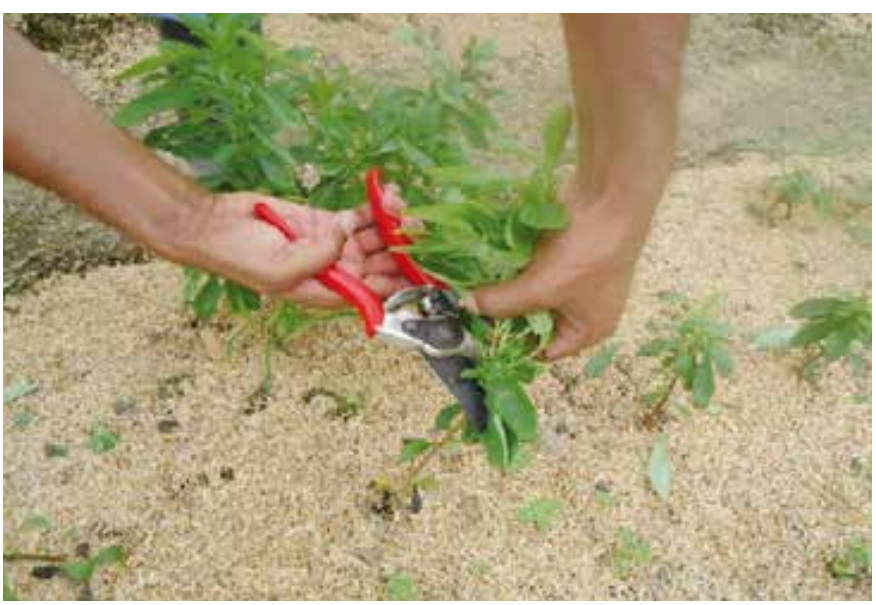

Foto $\mathrm{N}^{\circ} 7$. Cosecha de Stevia

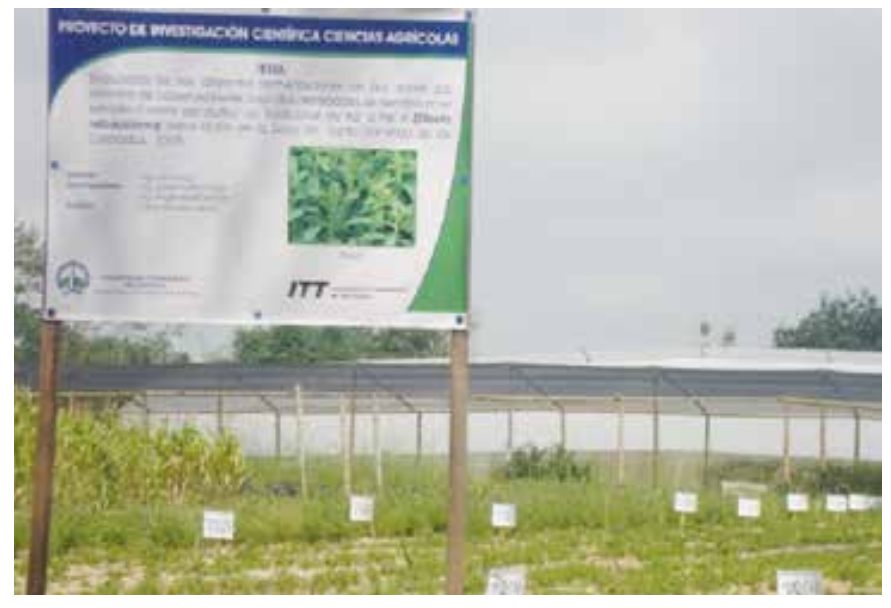

Foto $\mathrm{N}^{\circ} 6$. Desarrollo de cultivo Stevia

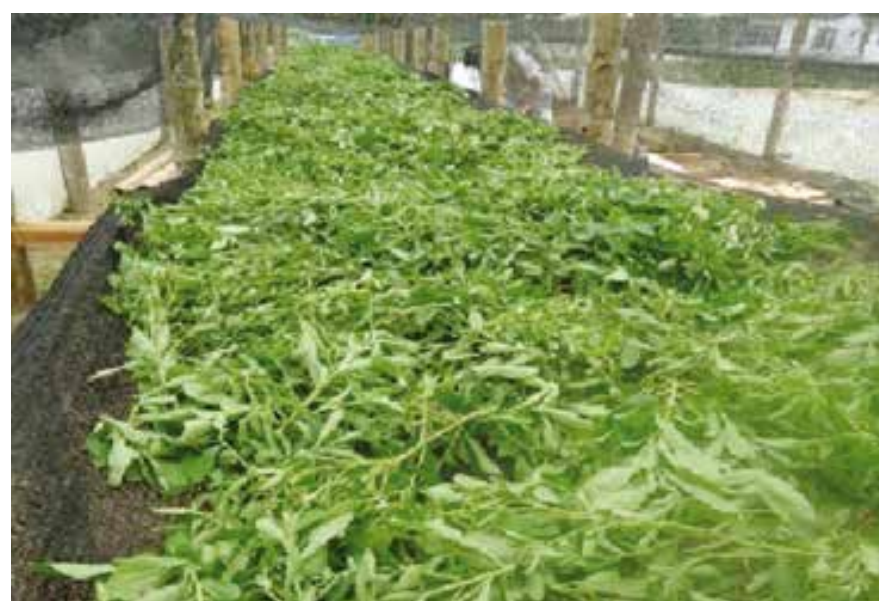

Foto $\mathrm{N}^{\circ} 8$. Secado de la hoja de Stevia 
